\title{
\begin{tabular}{l|l} 
Mibraries & DSpace@MIT
\end{tabular}
}

\author{
MIT Open Access Articles
}

\section{GALAXY CLUSTERS IN THE SWIFT /BURST ALERT TELESCOPE ERA: HARD X-RAYS IN THE INTRACLUSTER MEDIUM}

The MIT Faculty has made this article openly available. Please share how this access benefits you. Your story matters.

Citation: Ajello, M., P. Rebusco, N. Cappelluti, O. Reimer, H. Böhringer, J. Greiner, N. Gehrels, J. Tueller, and A. Moretti. “ GALAXY CLUSTERS IN THE SWIFT /BURST ALERT TELESCOPE ERA: HARD X-RAYS IN THE INTRACLUSTER MEDIUM ." The Astrophysical Journal 690, no. 1 (December 1, 2008): 367-388. (C) 2008 American Astronomical Society.

As Published: http://dx.doi.org/10.1088/0004-637x/690/1/367

Publisher: Institute of Physics/American Astronomical Society

Persistent URL: http://hdl.handle.net/1721.1/95915

Version: Final published version: final published article, as it appeared in a journal, conference proceedings, or other formally published context

Terms of Use: Article is made available in accordance with the publisher's policy and may be subject to US copyright law. Please refer to the publisher's site for terms of use. 


\title{
GALAXY CLUSTERS IN THE SWIFT/BURST ALERT TELESCOPE ERA: HARD X-RAYS IN THE INTRACLUSTER MEDIUM
}

\author{
M. Ajello ${ }^{1}$, P. Rebusco $^{2}$, N. Cappelluti ${ }^{1,3}$, O. Reimer ${ }^{4}$, H. Böhringer ${ }^{1}$, J. Greiner $^{1}$, N. Gehrels ${ }^{5}$, J. Tueller ${ }^{5}$, And \\ A. MORETTI $^{6}$ \\ ${ }^{1}$ Max Planck Institut für Extraterrestrische Physik, P.O. Box 1603, 85740, Garching, Germany; majello@mpe.mpg.de \\ ${ }^{2}$ Kavli Institute for Astrophysics and Space Research, MIT, Cambridge, MA 02139, USA \\ ${ }^{3}$ University of Maryland, Baltimore County, 1000 Hilltop Circle, Baltimore, MD 21250, USA \\ ${ }^{4}$ W.W. Hansen Experimental Physics Laboratory \& Kavli Institute for Particle Astrophysics and Cosmology, Stanford University, Palo Alto, CA, USA \\ ${ }_{5}$ Astrophysics Science Division, Mail Code 661, NASA Goddard Space Flight Center, Greenbelt, MD 20771, USA \\ ${ }^{6}$ INAF-OAB, via E. Bianchi 46, Merate (LC) 23807, Italy \\ Received 2008 May 1; accepted 2008 August 29; published 2008 December 1
}

\begin{abstract}
We report the detection of 10 clusters of galaxies in the ongoing Swift/Burst Alert Telescope (BAT) all-sky survey. This sample, which mostly comprises merging clusters, was serendipitously detected in the $15-55 \mathrm{keV}$ band. We use the BAT sample to investigate the presence of excess hard X-rays above the thermal emission. The BAT clusters do not show significant (e.g., $\geqslant 2 \sigma$ ) nonthermal hard X-ray emission. The only exception is represented by Perseus whose high-energy emission is likely due to NGC 1275. Using XMM-Newton, Swift/XRT, Chandra and BAT data, we are able to produce upper limits of the inverse Compton (IC) emission mechanism which are in disagreement with most of the previously-claimed hard X-ray excesses. The coupling of the X-ray upper limits of the IC mechanism to radio data shows that, in some clusters, the magnetic field might be larger than $0.5 \mu \mathrm{G}$. We also derive the first $\log N-\log S$ and luminosity function distributions of galaxy clusters above $15 \mathrm{keV}$.
\end{abstract}

Key words: acceleration of particles - galaxies: clusters: general - magnetic fields - radiation mechanisms: non-thermal - X-rays: general

Online-only material: color figures

\section{INTRODUCTION}

Galaxy clusters are potentially powerful observational probes of dark matter and dark energy. However, the use of clusters to measure cosmological parameters becomes accessible only when astrophysical uncertainties are well understood and controlled. Indeed, the nonthermal pressure due to cosmic rays (CRs), magnetic fields, and turbulence is a source of systematic bias when cluster masses are estimated using the assumption of hydrostatical equilibrium (e.g., Ensslin et al. 1997). The detection of clusters' $\mathrm{X}$-ray emission above $\sim 20 \mathrm{keV}$ is a fundamental step toward a firm grasp of these processes.

It is well understood that clusters of galaxies contain a large amount of hot gas, called intracluster medium (ICM), that comprises $10-15 \%$ of their total mass. The first X-ray observations already indicated the presence of this optically thin plasma, characterized by an atomic density of about $10^{-4}-10^{-2} \mathrm{~cm}^{-3}$ and temperatures of the order of $10^{7}-10^{8} \mathrm{~K}$ (e.g., Felten et al. 1966; Catura et al. 1972). Also, the fact that the observed X-ray radiation from clusters of galaxies is primarily due to the thermal bremsstrahlung emission of such diffuse hot plasma is well established (Sarazin 1988; Petrosian 2001).

However, evidence gathered at different wavelengths points to the existence of a nonthermal component. In particular, the detection of an extended synchrotron radio emission (e.g., Willson 1970; Harris \& Miley 1978; Giovannini et al. 1993; Giovannini \& Feretti 2000; Kempner \& Sarazin 2001; Thierbach et al. 2003) and, more recently, of a possibly nonthermal extreme ultraviolet (EUV) excess (Lieu et al. 1996; Bowyer et al. 1999; Bonamente et al. 2001; Durret et al. 2002) and soft excess (e.g., Werner et al. 2007) suggest the existence of a nonthermal X-ray component originating from a population of relativistic electrons. This scenario is confirmed by the detection of nonthermal emission in the hard X-ray spectra of a few galaxy clusters (see, e.g., Kaastra et al. 2008; Rephaeli et al. 2008, for a complete review). Still, its actual presence and origin remain controversial (Renaud et al. 2006a; Fusco-Femiano et al. 2007; Werner et al. 2007; Lutovinov et al. 2008).

A nonthermal component could arise from a population of point sources (e.g., active galactic nucleus (AGN) as in Katz 1976; Fabian et al. 1976; Fujita et al. 2007) or from inverse Compton (IC) scattering of cosmic microwave background (CMB) photons by relativistic electrons (e.g., Rephaeli 1979; Sarazin 1999). Other possible mechanisms are nonthermal bremsstrahlung (e.g., Sarazin 1999; Sarazin \& Kempner $2000)$ and synchrotron emission from ultrarelativistic electrons (Timokhin et al. 2004; Inoue et al. 2005; Eckert et al. 2008a). If the origin of the high-energy emission is IC scattering, then the presence of a large population of relativistic electrons (Lorentz factor $\gg 1000$ ) is required. This population could have been accelerated in shocks of different origin. Indeed, it could be associated with merger shocks (e.g., Fujita et al. 2003; Brunetti et al. 2004), dark matter bow shocks (e.g., Bykov et al. 2000), rampressure stripping of infalling galaxies (e.g., de Plaa et al. 2006), jets, AGN outbursts (Fujita et al. 2007, in the case of radio minihalos such as in Perseus cluster), and accretion shocks (e.g., Inoue et al. 2005). Nonthermal electrons lose energy on short timescales (below $1 \mathrm{Gyr}$ ). Therefore, some models consider a continued supply of primary accelerated electrons (i.e., via the first-order Fermi mechanism), while others assume a constant in situ reacceleration via CR collisions or the second-order Fermi mechanism.

If clusters are a large reservoir of nonthermal particles, then they should emit at higher energies, up to that of the $\gamma$-rays. 
Indeed, if CRs acceleration takes place at the shock fronts, then $\gamma$-rays can be produced via IC, nonthermal bremsstrahlung, and $\pi^{0}$ decay (e.g., Rephaeli 1979; Dar \& Shaviv 1995; Reimer et al. 2003, 2004; Blasi et al. 2007). A statistical upper limit on the flux above $100 \mathrm{MeV}$ was obtained by Reimer et al. (2003), analyzing the emission from 58 clusters observed with EGRET.

The role of CRs in the formation and evolution of clusters of galaxies has been much debated. Churazov et al. (2008) suggested that in massive galaxy clusters, hydrostatic equilibrium is satisfied reasonably well, as long as the source has not experienced a recent major merger. However, in nonrelaxed clusters the nonthermal pressure due to CRs, magnetic fields, and microturbulence can affect the mass estimates based on hydrostatic equilibrium (e.g., Miralda-Escude \& Babul 1995; Nagai et al. 2007). This would lead to a higher baryonic-to-total-mass ratio. Knowing the importance of CRs, the mechanisms that heat the ICM and the frequency at which it is shocked are crucial for the upcoming X-ray and Sunyaev-Zeldovich surveys (see Ando \& Nagai 2008).

In this paper, we report the Swift/Burst Alert Telescope (BAT) all-sky detection of 10 galaxy clusters in the $15-55 \mathrm{keV}$ band. This constitutes the first complete sample so far detected at these energies. We use this sample to investigate the role of nonthermal processes in clusters. The structure of the paper is the following. In Section 2, we describe the Swift/BAT observations and discuss the properties of each individual cluster (Section 2.2). In Section 3.1, we provide, for all the clusters, constraints on the nonthermal emission as well as an estimate of the clusters' magnetic fields (Section 3.3). The cluster source count distribution and the luminosity function are derived in Section 4. We discuss the results of our analysis in Section 5, while Section 6 summarizes our findings.

We adopt a Hubble constant of $H_{0}=70 \mathrm{~h}_{70} \mathrm{~km} \mathrm{~s}^{-1} \mathrm{Mpc}^{-1}$, $\Omega_{M}=0.3$, and $\Omega_{\Lambda}=0.7$. Unless otherwise stated, errors are quoted at the $90 \%$ confidence level (CL) for one interesting parameter and solar abundances are determined using the meteoritic values provided in Anders \& Grevesse (1989).

\section{THE BAT X-RAY SURVEY}

The BAT (Barthelmy et al. 2005), on board the Swift satellite (Gehrels et al. 2004), represents a major improvement in sensitivity for imaging of the hard X-ray sky. BAT is a coded mask telescope with a wide field-of-view (FOV; $120^{\circ} \times 90^{\circ}$ partially coded) aperture sensitive in the $15-200 \mathrm{keV}$ domain. BAT's main purpose is to locate Gamma-Ray Bursts (GRBs). While chasing new GRBs, BAT surveys the hard X-ray sky with an unprecedented sensitivity. Thanks to its wide FOV and its pointing strategy, BAT continuously monitors up to $80 \%$ of the sky every day. Results of the BAT survey (Markwardt et al. 2005; Ajello et al. 2008a) show that BAT reaches a sensitivity of $\sim 1 \mathrm{mCrab}$ in $1 \mathrm{Ms}$ of exposure. Given its sensitivity and the large exposure already accumulated in the whole sky, BAT is an excellent instrument for looking for the (faint) emission of galaxy clusters above $15 \mathrm{keV}$.

For the analysis presented here, we used all the available data taken from 2005 January to 2007 March. Since most of the cluster emission is expected to be thermal and thus rather soft, the chosen energy interval for the survey is $15-55 \mathrm{keV}$. The lower limit is dictated by the energy threshold of the detectors. The upper limit was chosen so as to avoid the presence of strong background lines, which could worsen the overall sensitivity. The data screening was performed according to Ajello et al. (2008a). The all-sky image is obtained as the weighted average of all the shorter observations. The average exposure time in our image is $3 \mathrm{Ms}, 1.3 \mathrm{Ms}$ and $5 \mathrm{Ms}$ being the minimum and maximum exposure times, respectively. The final image shows a Gaussian normal noise. Source candidates were identified as excesses above the $5 \sigma$ level. All these objects are then fitted with the BAT point spread function (PSF; using the standard BAT tool batcelldetect) to derive the best source position.

As shown in Ajello et al. (2008a), cross-correlating the BAT sources with the ROSAT All-Sky Survey Bright Source Catalogue (Voges et al. 1999) provides an easy and solid way to identify a large fraction $(\sim 70 \%)$ of them. Most of the uncorrelated sources are not present in the ROSAT survey because of absorption (either along the line of sight or intrinsic to the source). The unidentified sources are targeted by the Swift X-ray Telescope (XRT), which, in less than $10 \mathrm{ks}$, can pinpoint the exact counterpart (e.g., Tueller et al. 2005a, 2005b; Kennea et al. 2005).

The details about the complete source list will be given in an upcoming publication. Here we report on the detection of galaxy clusters above $5 \sigma$ in the $15-55 \mathrm{keV}$ band.

\subsection{Clusters' Identification}

Identifying clusters of galaxies as counterparts of BAT objects is not a straightforward process. Indeed, coded mask telescopes are rather insensitive to diffuse sources, which extend over angles much larger than the projection of the mask tile on the sky (i.e., a few tens of arcminutes for BAT). Even though procedures exist to quantify the extent of diffuse sources in coded mask instruments (see Renaud et al. 2006a, 2006b; Lutovinov et al. 2008, for the case of the Coma cluster), their application is limited only to high signal-to-noise $(\mathrm{S} / \mathrm{N})$ objects. Given the extent of the BAT PSF $\left(22^{\prime}\right)$, Coma is the only object whose emission is clearly extended in our investigation. Thus, for all other objects, the morphology of the source cannot be used to understand whether the BAT source is associated with the cluster or only with its brightest AGN. We therefore performed a spectral analysis (see Section 2.2) of those BAT sources that are spatially associated with galaxy clusters. All sources presented here show a significant thermal component that we interpret as thermal bremsstrahlung from the ICM, and thus are securely associated with the proposed clusters. Our sample contains 10 galaxy clusters. Table 1 reports the position, significance, total exposure time, and other details of all the detected clusters.

\subsection{Spectral Analysis}

For each galaxy cluster, we extracted a $15-195 \mathrm{keV}$ spectrum with the method described in Ajello et al. (2008c). Here, we recall the main steps; the details can be found in the aforementioned paper. For a given source, we extract a spectrum from each observation where the source is in the FOV. These spectra are corrected for residual background contamination and for vignetting; the per-pointing spectra are then (weighted) averaged to produce the final source spectrum. Thus, the final spectrum represents the average source emission over the time span considered here (2.5 years). The accuracy of these spectra is discussed in Section 2.3.

For all the clusters, we extracted a $0.3-10 \mathrm{keV}$ spectrum using archival observations of XMM-Newton, Chandra, and Swift/ XRT. Considering that for BAT, all clusters, except Coma, are point-like objects, we extracted (unless otherwise stated) all cluster photons within $10^{\prime}$ from the position of the BAT centroid. In most cases, this selection allows us to include most of the 
Table 1

Clusters Detected in the $15-55 \mathrm{keV}$ Band

\begin{tabular}{lccrcccc}
\hline \hline Name & $\begin{array}{c}\text { R.A. } \\
(\mathrm{J} 2000)\end{array}$ & $\begin{array}{c}\text { Decl. } \\
(\mathrm{J} 2000)\end{array}$ & $\mathrm{S} / \mathrm{N}$ & ID & $z$ & $\begin{array}{c}\text { Exposure } \\
(\mathrm{Ms})\end{array}$ & $\begin{array}{c}\text { Offset } \\
(\operatorname{arcmin})\end{array}$ \\
\hline J0319.8+4130 & 49.9573 & 41.5110 & 28.00 & Perseus & 0.0175 & 2.89 & 0.5 \\
J0431.3-6126 & 67.8297 & -61.4388 & 5.61 & A3266 & 0.0590 & 3.81 & 2.1 \\
J0908.9-0938 & 137.2391 & -9.6346 & 8.28 & A0754 & 0.0530 & 2.96 & 1.8 \\
J1259.4+2757 & 194.8531 & 27.9523 & 19.95 & Coma cluster & 0.0230 & 4.32 & 5.1 \\
J1347.7-3253 & 206.9500 & -32.9000 & 5.05 & A3571 & 0.0397 & 1.78 & 4.5 \\
J1511.0+0544 & 227.7500 & 5.7485 & 5.33 & A2029 & 0.0770 & 2.71 & 0.8 \\
J1558.5+2714 & 239.6256 & 27.2417 & 7.11 & A2142 & 0.0890 & 3.62 & 3.3 \\
J1638.8-6424 & 249.7136 & -64.4000 & 6.90 & Triangulum A & 0.0510 & 1.77 & 4.9 \\
J1712.3-2319 & 258.0914 & -23.3242 & 21.63 & Ophiucus & 0.028 & 1.30 & 1.7 \\
J1920.9+4357 & 290.2405 & 43.9646 & 11.72 & A2319 & 0.056 & 3.87 & 2.2
\end{tabular}
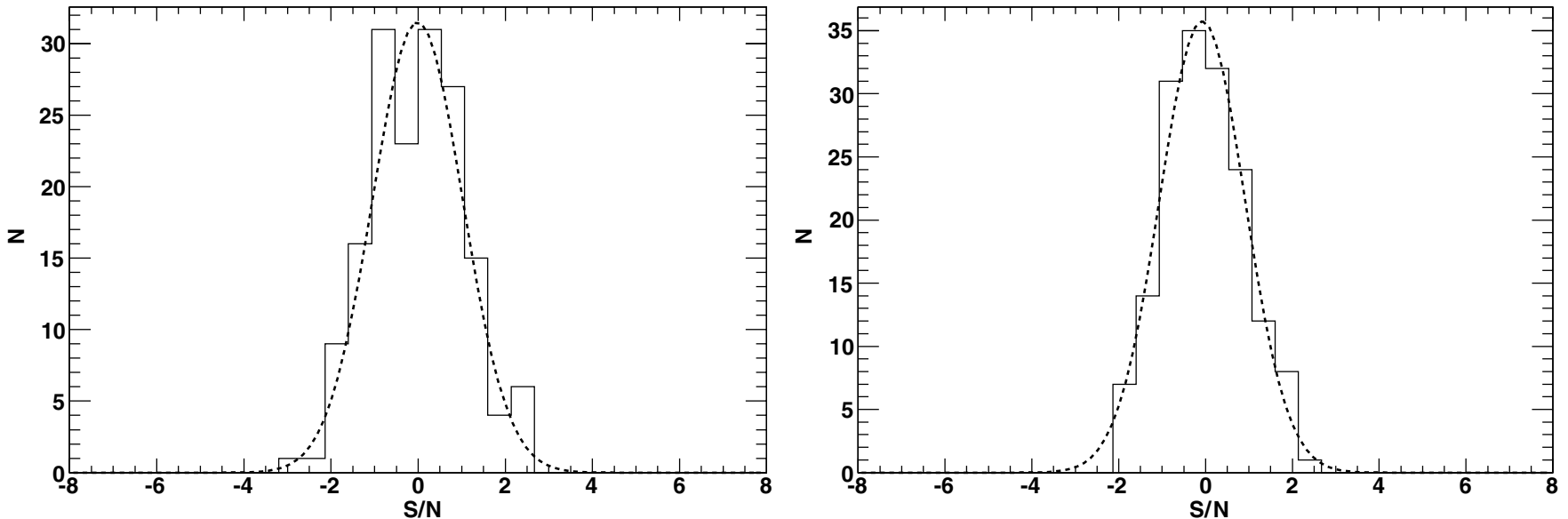

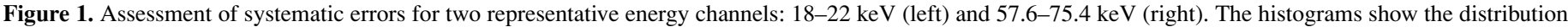

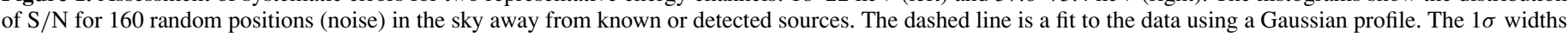
of the Gaussian profiles are compatible with 1.0.

emission of the cluster. For those cases where there is clearly emission outside of our selection region, ${ }^{7}$ we accounted for the missed flux using the beta profiles available in the literature. The details are given in the case-by-case section (Section 2.4). The level of the background was evaluated in regions of the CCDs not contaminated by the cluster emission or using blank-sky observations (e.g., Lumb et al. 2002; Read \& Ponman 2003). In all cases, we considered the systematic uncertainty connected to the background subtraction, in the $0.3-10 \mathrm{keV}$ band, to be $2 \%$. All spectra were rebinned in order to have a minimum of 50 counts $(\geqslant 7 \sigma)$ per bin.

As a standard procedure, we started fitting all the spectra with the most simple and plausible spectral model. In all cases, this was a single-temperature thermal model with absorption fixed at the Galactic value. Only when the value of the $\chi^{2} /$ degrees of freedom (dof) was greater than 1 did we try to add a second thermal model or a power law. In this case, we chose the model that produced the best improvement in the fit (evaluated using the $f$-test) and the best residuals.

Various authors have reported detection of hard X-ray excesses for some of the clusters present in our sample. For those cases where we do not directly detect such components, we tested whether our data are consistent with the reported nonthermal hard X-ray emission. This was done by adding a power law to the thermal model used. We fixed the power-law index to 2.0, which is a value generally accepted for the nonthermal hard

\footnotetext{
7 In some cases, the extent of the selection region is limited by the size of the
} CCD.
X-ray component generated by IC of relativistic electrons off CMB photons (e.g., Reimer et al. 2004; Nevalainen et al. 2004). We then let the power-law normalization vary until the $\Delta \chi^{2}$ increment was larger than 2.7 (6.64). According to Avni (1976), this gives the $90 \%$ (99\%) CL on the parameter of interest. This allows us to investigate the level of nonthermal flux, which is consistent with our data.

\subsection{Accuracy of BAT Spectra}

When dealing with spectral features that are at the limiting sensitivity of a given instrument, it is important to make sure that all systematic uncertainties have been carefully taken care of. In order to test the reliability of our spectral extraction method, we extracted more than 160 spectra at random positions in the sky at least $30^{\prime}$ away from the potential (or detected) X-ray sources reported in the International Gamma-Ray Astrophysics Laboratory (INTEGRAL) reference catalog (Ebisawa et al. 2003). The mean (raw) exposure of our spectral sample is 4.6 Ms. In each energy channel, the average flux is consistent within $1 \sigma$ with zero as expected for pure noise and for efficient background subtraction. Moreover, the $\mathrm{S} / \mathrm{N}$ distributions (i.e., flux divided by its error in a given energy channel; examples are shown in Figure 1) are all consistent with normal Gaussian distributions. Both findings show that our spectra can be trusted in the whole energy range (15-200 keV) and that uncertainties are well estimated.

Moreover, we can use the randomly extracted spectra to measure the average spectral sensitivity of BAT in a given energy 


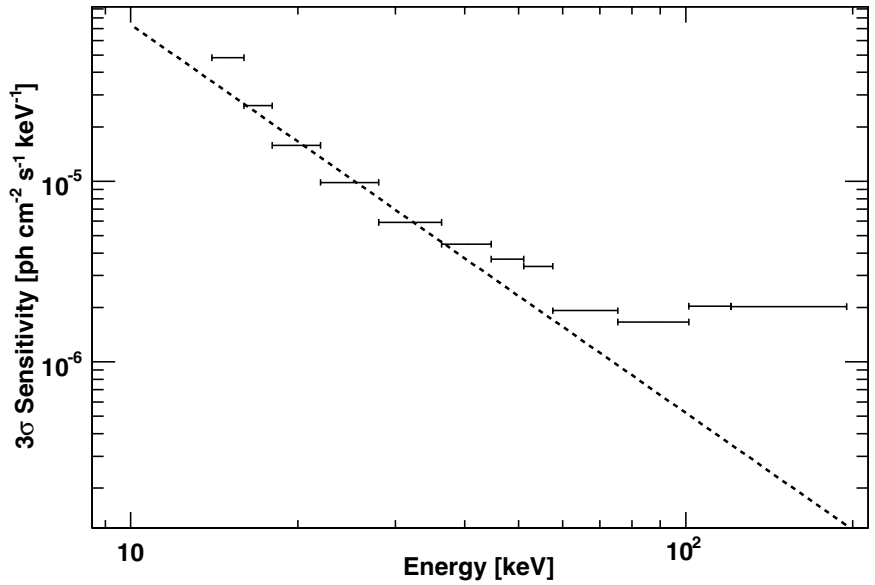

Figure 2. $3 \sigma$ average spectral sensitivity as a function of energy based on the analysis of 160 randomly extracted spectra. The dashed line is the Crab Nebula spectrum divided by 1000 .

channel. This is done by deriving the standard deviation of the flux distribution for each energy channel. As shown in Figure 2, the $3 \sigma$ sensitivity in each energy channel is very close to $1 \mathrm{mCrab}$, except above $100 \mathrm{keV}$.

\subsection{Individual Cluster Analysis}

\subsubsection{Perseus}

Swift J0319.8+4130 is certainly associated with the Perseus cluster (A426). The BAT detection (see Figure 3) is well centered on the cluster. Perseus is one of the most studied galaxy clusters and its detection in X-rays dates back to the seventies (Fritz et al. 1971; Forman et al. 1972). XMM-Newton observations (Churazov et al. 2003) showed that the central region is contaminated by the emission of the AGN hosted by the brightest galaxy in Perseus, NGC 1275. A hard X-ray component has been detected with $H E A O 1$ by Primini et al. (1981). Nevalainen et al. (2004) used BeppoSAX and previous Rossi X-Ray Timing Explorer ( $R X T E$ ) measurements to prove that this nonthermal component is variable and must therefore be connected to the central bright AGN. Sanders \& Fabian
(2007) reported, using Chandra, the presence of nonthermal $\mathrm{X}$-ray emission in the core of Perseus in correspondence to the radio mini-halo (Gisler \& Miley 1979; Gitti et al. 2002). This nonthermal emission, which displays a power-law behavior with a photon index of 2.0, seems to exceed the flux of NGC 1275 by a factor of $\sim 3$ (Sanders et al. 2004).

The BAT spectrum shows evidences of a hard X-ray excess. Indeed, it can be fitted by a steep power law (photon index of $3.5 \pm 0.1$ and $\chi_{\text {red }}^{2}=2.3$ ) while it rejects a simple bremsstrahlung fit $\left(\chi_{\text {red }}^{2}=3.6\right)$. The fit improves $\left(\chi_{\text {red }}^{2}=1.50\right)$ if we use a composite model, the sum of the (bremsstrahlunglike) gas emission and the (power-law-like) AGN emission. The improvement of the fit is statistically significant as confirmed by the $f$-test probability of $1.2 \times 10^{-2}$. The best-fit temperature is $6.4_{-2.3}^{+2.3} \mathrm{keV}$ and the photon index is $2.5_{-1.0}^{+1.9}$. If we fix the photon index at the value (1.65) determined by Churazov et al. (2003), we derive an extrapolated $0.5-8.0 \mathrm{keV}$ luminosity of $\sim 0.4 \times 10^{42} \mathrm{erg} \mathrm{s}^{-1}$, which is in agreement with the luminosity measured by XMM-Newton. This supports the idea that the hardtail seen in the BAT spectrum is due to NGC 1275 and not due to a nonthermal component originating in the ICM. Moreover, if we extrapolate, using a power law with a photon index of 2.0, the nonthermal flux found in the $2-10 \mathrm{keV}$ range by Sanders et al. (2005) to the $50-100 \mathrm{keV}$ band, we get a value of $2.7 \times 10^{-11} \mathrm{erg} \mathrm{cm}^{-2} \mathrm{~s}^{-1}$. This flux is a factor of $\sim 4$ larger than the total cluster flux observed by BAT in the same energy band. Recently, Molendi \& Gastaldello (2008) analyzed a long XMM-Newton observation and did not find evidence for nonthermal emission. According to them, the discrepancy between the Chandra and XMM-Newton results was due to a problem in the effective area calibration of Chandra.

An XRT observation of 5.4 ks was carried out in 2007 July. Given the size of the XRT CCD, we extracted all source photons within $6^{\prime}$ from the BAT centroid. The surface-brightness profile of Perseus is best described by the sum of a power law and a beta model. Adopting this model, as suggested by Ettori et al. (1998), yields that $\sim 94 \%$ of the total cluster emission falls within our selection. The joint XRT-BAT spectrum can be fitted by a sum of two Astrophysical Plasma Emission Code (APEC; Smith et al. 2001) models and a power law. The low-temperature
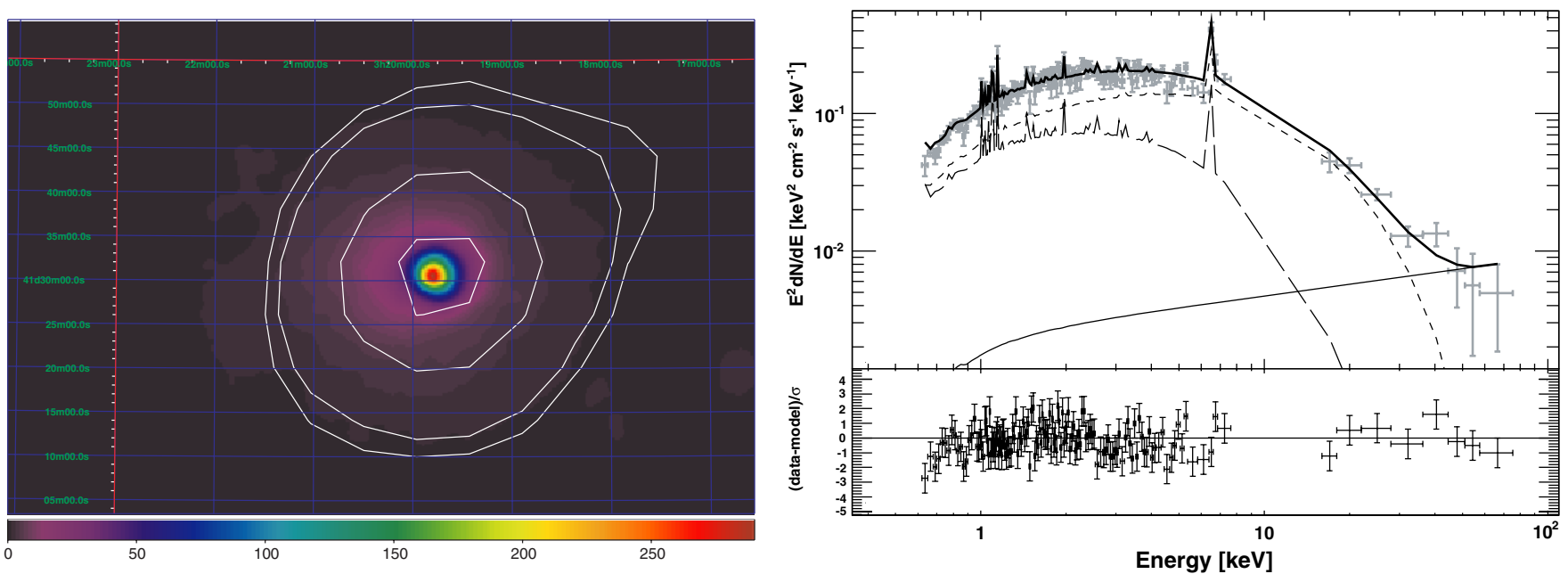

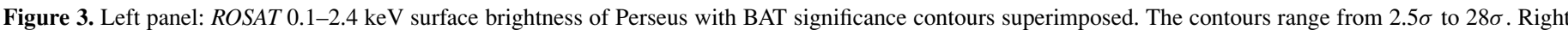

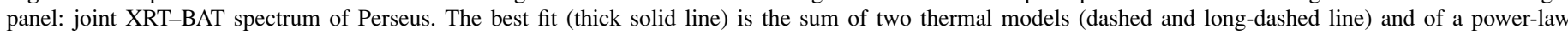
component (thin solid line).

(A color version of this figure is available in the online journal.) 

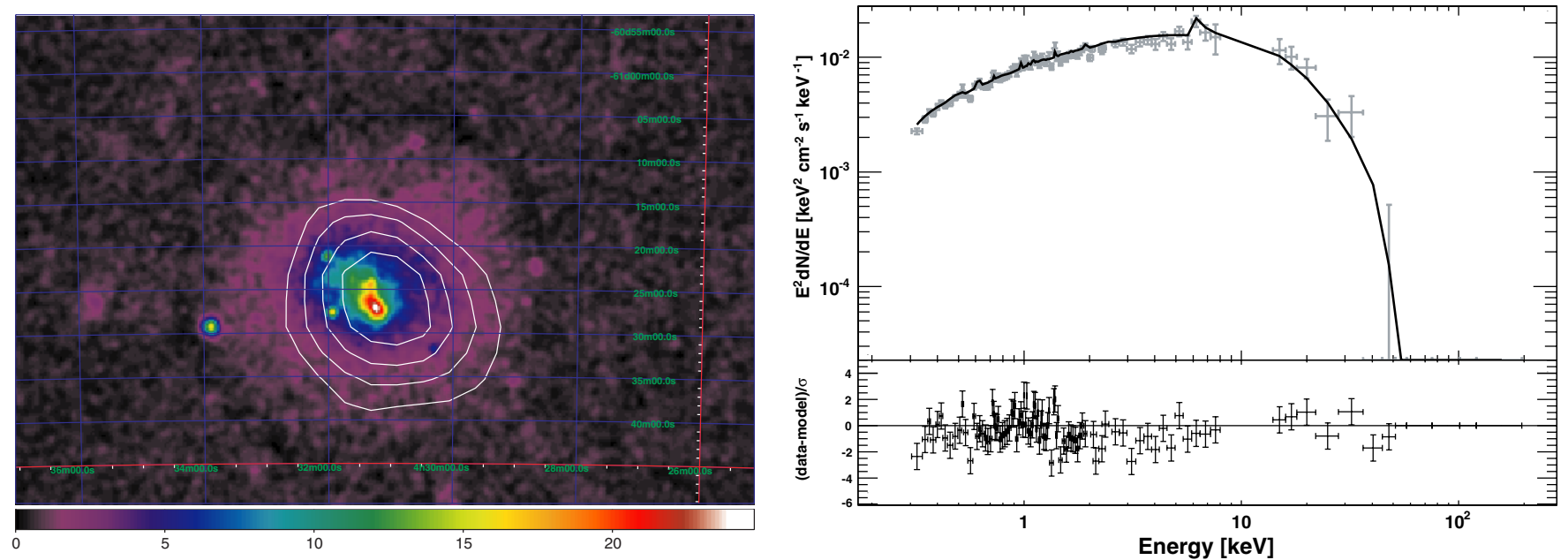

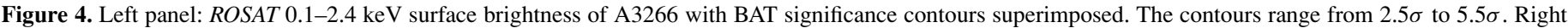
panel: joint fit to XMM-Newton-BAT data for A3266 with a thermal model. The best model is shown as a solid line.

(A color version of this figure is available in the online journal.)

component, which accounts for the cool core of the cluster, has a temperature of $3.0_{-0.7}^{+0.4} \mathrm{keV}$ and an abundance of $0.43_{-0.16}^{+0.20}$ solar. The warmer component displays a temperature of $6.40_{-0.71}^{+0.62} \mathrm{keV}$ and an abundance of $0.31_{-0.15}^{+0.15}$ solar. These results are in line with the analyses of Churazov et al. (2003) and Sanders et al. (2005). Both the power-law photon index of $1.7_{-0.7}^{+0.3}$ and the luminosity in the $0.5-8.0 \mathrm{keV}$ band of $\sim 8 \times 10^{-42} \mathrm{erg} \mathrm{s}^{-1}$ are compatible with the values found for NGC 1275 by Churazov et al. (2003) and those determined in the next section. The photon index is slightly harder than the average photon index (2.0) of BAT AGN; however it is not unusual for radio-loud objects (e.g., Ajello et al. 2008c).

\subsubsection{The Nucleus of Perseus}

In order to study the nuclear emission in more details, we analyzed a $125 \mathrm{ks}$ long XMM-Newton observation (observation 0305780101). We extracted the spectrum of the nucleus in a radius of $25^{\prime \prime}$ and evaluated the local background in an annulus around the source region. We note that the results presented here are not sensitive to the radius of the extraction region if this is in the $10^{\prime \prime}-30^{\prime \prime}$ range. The $0.2-9.0 \mathrm{keV}$ spectrum of the nucleus is well fitted $\left(\chi^{2} /\right.$ dof $\left.=960.1 / 731\right)$ by an absorbed power-law model with absorption consistent with the Galactic one and a photon index of $1.60 \pm 0.02$. Moreover, we find evidence (at the $95 \% \mathrm{CL}$ ) of a $\mathrm{K} \alpha$ iron line with an equivalent width of $90.2 \pm 45.0 \mathrm{eV}$. An absorbed APEC model with a temperature of $12.6 \pm 0.7 \mathrm{keV}$ provides the worst fit $\left(\chi^{2} /\right.$ dof $\left.=1167.1 / 732\right)$ to the data. In particular, the absorption would be required to be lower than the Galactic one at $99 \%$ CL. This fact, in conjunction with the presence of the iron line, supports the evidence that the nonthermal emission in the nucleus of the Perseus cluster is produced by the central AGN. The nonthermal luminosities in the $0.5-8.0 \mathrm{keV}$ and $2.0-10.0 \mathrm{keV}$ bands are $7.6_{-0.2}^{+0.2} \times 10^{42} \mathrm{erg} \mathrm{s}^{-1}$ and $6.5_{-0.2}^{+0.2} \times 10^{42} \mathrm{erg} \mathrm{s}^{-1}$, respectively. In order to check these results, we extracted a similar spectrum of the nucleus using Swift/XRT data and selected an extraction region of $10^{\prime \prime}$. The XRT data are compatible with those of XMM-Newton. Indeed, fixing the absorption at the Galactic value, we find that the XRT data are compatible with a power-law model with a photon index of $1.6 \pm 0.1$ and that the $2.0-10.0 \mathrm{keV}$ luminosity is
$8.2_{-1.0}^{+1.1} \times 10^{42} \mathrm{erg} \mathrm{s}^{-1}$. Thus, the nucleus displays a moderate variability between the XMM-Newton and Swift/XRT observation epochs. This supports, once more, the interpretation that the nonthermal emission is produced by the central AGN.

\subsection{3. $A 3266$}

Swift J0431.3-6126 is associated with A3266. Figure 4 shows that the BAT source is well centered on the cluster emission as seen by ROSAT. A3266 (also known as Sersic 40-6) was first detected in X-rays by the Uhuru satellite (Giacconi et al. 1972). According to many authors (e.g., Sauvageot et al. 2005; Finoguenov et al. 2006, and references therein), A3266 recently underwent a major merger, probably with a subcluster that was stripped during the encounter with the A3266 dense core. De Grandi \& Molendi (1999) and Nevalainen et al. (2004) observed A3266 with BeppoSAX. The first group modeled the BeppoSAX broad-band spectrum (2-50 keV) with a simple, optically thin, thermal emission model at the temperature of $8.1 \pm 0.2 \mathrm{keV}$, while the second group found marginal evidence $(0.8 \sigma)$ of nonthermal X-ray excess.

The BAT spectrum, shown in Figure 4, is consistent with the findings of De Grandi \& Molendi (1999). A bremsstrahlung model with a plasma temperature of $6.9_{-1.8}^{+2.5} \mathrm{keV}$ indeed provides a good fit to the data $\left(\chi^{2} /\right.$ dof $\left.=7 \cdot 2 / 10\right)$. XMM-Newton observed A3266 for $8.6 \mathrm{ks}$ in 2000 September. The cluster is not centered on the EPIC-PN CCD. Thus, we could extract only photons within a circular region of $\sim 8^{\prime}$ radius centered on the BAT centroid. In order to estimate the flux missed by our selection, we adopt, for the cluster surface brightness, a beta profile with $\beta=0.51$ and core radius $R_{c}=3^{\prime} .1$ (Sauvageot et al. 2005). According to our estimate, $80 \%$ of the total cluster flux is contained in our selection. Therefore, when jointly fitting the XMM-Newton and the BAT data, we use such a cross-normalization factor. The combined $X M M$ Newton-BAT spectrum is well fitted by a single APEC model with a plasma temperature of $8.0_{-0.4}^{+0.4} \mathrm{keV}$ and $0.41_{-0.13}^{+0.13}$ solar abundance. We derive a $99 \% \mathrm{CL}$ on the nonthermal $50-100 \mathrm{keV}$ flux of $5.70 \times 10^{-13} \mathrm{erg} \mathrm{cm}^{-2} \mathrm{~s}^{-1}$.

Extended radio emission correlated with A3266 has been reported (Robertson \& Roach 1990; Brown \& Burns 1991). In 
Table 2

Spectral Parameters from Combined XMM-Newton/XRT/Chandra and BAT Fits (Errors are 90\% CL)

\begin{tabular}{|c|c|c|c|c|c|c|}
\hline Name & $\begin{array}{c}\text { Flux }^{\mathrm{a}} \\
\left(10^{-11} \mathrm{cgs}\right)\end{array}$ & $\begin{array}{c}L_{\mathrm{x}}{ }^{\mathrm{a}} \\
\left(10^{43} \mathrm{erg} \mathrm{s}^{-1}\right) \\
\end{array}$ & $\begin{array}{c}\mathrm{kT} \\
(\mathrm{keV}) \\
\end{array}$ & $\Gamma$ & Model & $\chi^{2} /$ dof \\
\hline Perseus & $3.90_{-1.65}^{+0.10}$ & $2.7_{-1.1}^{+0.1}$ & $3.00_{-0.71}^{+0.40} / 6.40_{-0.71}^{+0.62}$ & $1.7_{-0.7}^{+0.3}$ & apec+apec+pow & $152.8 / 144$ \\
\hline A3266 & $0.73_{-0.11}^{+0.10}$ & $6.9_{-0.9}^{+0.9}$ & $8.0_{-0.4}^{+0.4}$ & & apec & $666.8 / 841$ \\
\hline A0754 & $1.11_{-0.04}^{+0.04}$ & $8.3_{-0.3}^{+0.3}$ & $9.3_{-0.4}^{+0.4}$ & & apec+pow & $1217.0 / 1072$ \\
\hline Coma $^{b}$ & $2.33_{-0.22}^{+0.23}$ & $3.0_{-0.4}^{+0.2}$ & $8.40_{-0.24}^{+0.25} / 1.45_{-0.11}^{+0.21}$ & & apec+apec & $846.5 / 856$ \\
\hline A3571 & $0.63_{-0.06}^{+0.09}$ & $2.7_{-0.4}^{+0.3}$ & $6.0_{-0.2}^{+0.2}$ & & apec & $723.9 / 1367$ \\
\hline A2029 & $1.01_{-0.45}^{+0.16}$ & $16.8_{-4.7}^{+2.4}$ & $4.1_{-1.5}^{+1.7} / 9.6_{-2.0}^{+2.0}$ & & apec+apec & $394.2 / 363$ \\
\hline A2142 & $0.90_{-0.10}^{+0.10}$ & $21.5_{-2.6}^{+3.5}$ & $8.40_{-0.45}^{+0.64}$ & & apec & $361.9 / 398$ \\
\hline Triangulum A & $1.30_{-0.10}^{+0.10}$ & $8.8_{-0.2}^{+0.6}$ & $9.30_{-0.30}^{+0.30}$ & & apec & $925.8 / 1074$ \\
\hline Ophiucus & $5.7_{-0.5}^{+0.5}$ & $9.38_{-0.14}^{+0.28}$ & $9.93_{-0.24}^{+0.24}$ & & apec & $323.1 / 351$ \\
\hline A2319 & $1.56_{-0.14}^{+0.14}$ & $13.0_{-0.8}^{+0.9}$ & $9.23_{-0.27}^{+0.27}$ & & apec & $1151.3 / 1274$ \\
\hline
\end{tabular}

Notes.

${ }^{\text {a }}$ Flux and luminosities are computed in the $15-55 \mathrm{keV}$ band.

$\mathrm{b}$ The spectral values reported for Coma are only representative for the source extraction region (i.e., 10' around the BAT centroid; see Section for more details).

Table 3

$3 \sigma$ Upper Limits on the Nonthermal Component and Clusters' Properties

\begin{tabular}{|c|c|c|c|c|c|c|c|c|}
\hline NAME & $\mathrm{CC}^{\mathrm{a}} ?$ & Merger? & $\begin{array}{c}F_{50-100 \mathrm{keV}^{\mathrm{b}}} \\
\left(10^{-12} \mathrm{erg} \mathrm{cm}^{-2} \mathrm{~s}^{-1}\right)\end{array}$ & $\begin{array}{c}B \\
(\mu \mathrm{G})\end{array}$ & $\underset{(\mathrm{Jy})}{S_{\text {radio }}}$ & $\begin{array}{l}v_{\text {radio }} \\
(\mathrm{MHz})\end{array}$ & $\alpha$ & $\operatorname{Ref}^{c}$ \\
\hline Perseus & $\mathrm{y}$ & $\mathrm{y}$ & $\ldots$ & $\ldots$ & $\ldots$ & $\ldots$ & $\ldots$ & $\ldots$ \\
\hline A0754 & $\mathrm{n}$ & $\mathrm{y}$ & $<6.50$ & $>0.10$ & 0.086 & 1365 & 1.5 & 2 \\
\hline Coma & $\mathrm{n}$ & $\mathrm{y}$ & $\ldots$ & $\ldots$ & $\ldots$ & $\ldots$ & $\ldots$ & $\ldots$ \\
\hline A3571 & $\mathrm{y}^{\mathrm{d}}$ & $\mathrm{n}^{\mathrm{e}}$ & $<11.5$ & $>0.03$ & 0.0084 & 1380 & $1.5^{\mathrm{g}}$ & 3 \\
\hline A2142 & $y^{f}$ & $\mathrm{y}$ & $<5.35$ & $>0.06$ & 0.0183 & 1400 & $1.5^{\mathrm{g}}$ & 5 \\
\hline Triangulum A & $y^{f}$ & $y^{f}$ & $<4.65$ & $>0.17$ & $<0.033$ & 4850 & $1.5^{\mathrm{g}}$ & 6 \\
\hline Ophiucus & $\mathrm{n}$ & $\mathrm{n}$ & $<5.89$ & $>0.11$ & 6.4 & 160 & 2.0 & 7 \\
\hline A2319 & $y^{d}$ & $\mathrm{y}$ & $<3.41$ & $>0.10$ & 1.0 & 610 & 0.92 & 8 \\
\hline
\end{tabular}

Notes.

a $\mathrm{CC}=$ Cool Core.

b BAT data alone were used to estimate the upper limits.

c References for the radio flux.

d Moderate CC.

e The morphology and temperature map indicate that it is a relaxed cluster, but the radio structure points at late stages of merging.

${ }^{\mathrm{f}}$ Under discussion.

g Arbitrary spectral index.

References. (1) Brown \& Burns 1991; (2) Fusco-Femiano et al. 2003; (3) Condon et al. 1998; (4) Giovannini \& Feretti 2000;

(5) Condon et al. 1993; (6) Slee 1977; (7) Feretti et al. 1997.

order to estimate the magnetic field (see Section 3.1 and Table 3), we adopt the radio data from Brown \& Burns (1991), based on the Parkes catalogue, namely a flux density $S_{2700 \mathrm{MHz}}=1.070 \mathrm{Jy}$ and a spectral index $\alpha=0.95$.

\subsection{4. $A 0754$}

Swift J0908.9 - 0938 is associated with the well-studied cluster of galaxies A0754. X-ray maps indicate that A0754 is far from hydrostatic equilibrium, experiencing a violent merger (Henry \& Briel 1995; Henriksen \& Markevitch 1996). Its detection by RXTE (Valinia et al. 1999; Revnivtsev et al. 2004) and BeppoSAX (Fusco-Femiano et al. 2003) above $15 \mathrm{keV}$ make the association of the cluster with the BAT source secure. While the Rossi X-Ray Timing Explorer (RXTE) detections do not measure any significant hard X-ray excess, BeppoSAX detects a hard tail with a significant deviation from the thermal component in the $50-70 \mathrm{keV}$ energy range. It is worth noting that the BAT centroid ${ }^{8}$ falls $\sim 6^{\prime}$ western of the brightest region of the cluster (see Figure 5). Chandra analysis of the gas temperature spatial distribution indeed shows that the BAT position corresponds to regions of hot $(T \approx 10-15 \mathrm{keV})$ gas (Markevitch et al. 2003). The analysis of XMM-Newton data confirms the existence of hot regions in the western part of the cluster (Henry et al. 2004). On the other hand, centroid shifts as a function of the waveband are a common indication of a merging cluster (O'Hara et al. 2004).

The BAT spectrum, shown in Figure 5, is well fitted $\left(\chi^{2} /\right.$ dof $\left.=6.3 / 9\right)$ by a single bremsstrahlung model with a plasma temperature of $9.9_{-2.6}^{+4.3} \mathrm{keV}$. This is in good agreement with the temperature of $9.4_{-0.17}^{+0.16} \mathrm{keV}$ reported by Fusco-Femiano et al. (2003), and 9.0 $\pm 0.13 \mathrm{keV}$ reported by Valinia et al. (1999). The BeppoSAX 10-40 keV nonthermal flux of $\sim 1.6 \times 10^{-12} \mathrm{erg}$

\footnotetext{
8 For an $8 \sigma$ detection, the expected maximum offset of the BAT centroid is $\sim 2.5$ (see Figure 10 in Ajello et al. 2008a).
} 

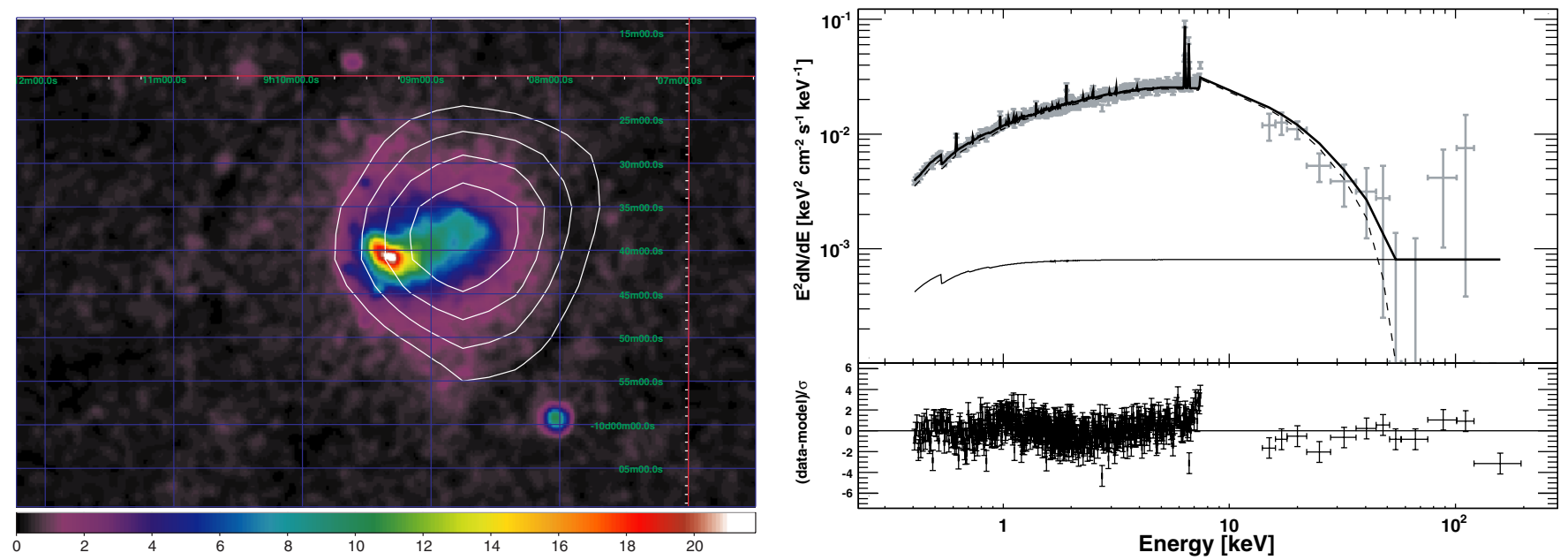

Figure 5. Left panel: ROSAT 0.1-2.4 keV surface brightness of A0754 with BAT significance contours superimposed. The contours range from $2.5 \sigma$ to $8.0 \sigma$. Right panel: joint fit to XMM-Newton-BAT data. The best-fit model (thick solid line) is the sum of a thermal model (dashed line) and of a power law (thin solid line). (A color version of this figure is available in the online journal.)

Table 4

Nonthermal Emission from Combined XMM-Newton/XRT/Chandra and BAT Data.

\begin{tabular}{lcc}
\hline \hline Name & $\begin{array}{c}F_{50-100 \mathrm{keV}^{\mathrm{a}}} \\
\left(10^{-12} \mathrm{erg} \mathrm{cm}^{2} \mathrm{~s}^{-1}\right)\end{array}$ & $\begin{array}{c}B^{\mathrm{b}} \\
(\mu \mathrm{G})\end{array}$ \\
\hline Perseus & $\ldots$ & $\ldots$ \\
A3266 & $<0.57$ & $>0.55$ \\
A0754 & $\ldots$ & $\ldots$ \\
Coma & $\ldots$ & $\ldots$ \\
A3571 & $1.4_{-0.4}^{+0.4}$ & $\sim 0.08$ \\
A2029 & $<1.27$ & $>0.42$ \\
A2142 & $<1.50$ & $>0.10$ \\
Triangulum Australis & $<0.65$ & $>0.39$ \\
Ophiucus & $<2.80$ & $>0.15$ \\
A2319 & $<0.67$ & $>0.15$ \\
\hline
\end{tabular}

\section{Notes.}

a The flux has been estimated using a power-law spectrum with a photon index of 2.0 in the 1-200 keV energy band. Upper limits are $99 \%$ CL while errors are $90 \%$ CL.

b In order to compute the intensity of the magnetic field, we used the same radio data reported in Table 3.

$\mathrm{cm}^{-2} \mathrm{~s}^{-1}$ is consistent with the $(90 \%)$ upper limit from BAT of $6.5 \times 10^{-12} \mathrm{erg} \mathrm{cm}^{-2} \mathrm{~s}^{-1}$.

XMM-Newton observed A0754 for $11 \mathrm{ks}$ in 2001 May. The $X M M-N e w t o n-B A T$ data are well fitted by a single APEC model with a plasma temperature of $8.5_{-0.13}^{+0.19} \mathrm{keV}$ and $0.29 \pm 0.03$ solar abundance. Adding a power-law model, with the photon index fixed to 2.0 , improves the fit $\left(f\right.$-test probability $\left.4.6 \times 10^{-9}\right)$. The best-fit temperature is $9.3 \pm 0.4 \mathrm{keV}$ and the nonthermal 50 $100 \mathrm{keV}$ flux is $7.6_{-2.7}^{+2.4} \times 10^{-13} \mathrm{erg} \mathrm{cm}^{-2} \mathrm{~s}^{-1}$. The nonthermal flux in the $10-40 \mathrm{keV}$ band is $1.7_{-0.6}^{+0.2} \times 10^{-12} \mathrm{erg} \mathrm{cm}^{-2} \mathrm{~s}^{-1}$ and is in good agreement with the nonthermal flux measured by Fusco-Femiano et al. (2003). However, Fusco-Femiano et al. (2003) also discussed the possibility that the nonthermal flux will be produced by the BL Lac object 26W20. This object lies $\sim 24^{\prime}$ away from the BAT centroid and outside the XMMNewton FOV; thus, we can rule out that it contributes to the detected nonthermal flux.

However, we note that several point-like objects appear in the $X M M-N e w t o n$ image and within $10^{\prime}$ from the BAT centroid. A simple hardness ratio analysis reveals that the hardest object is located at R.A. $=09^{\mathrm{h}} 09^{\mathrm{m}} 13.7$, Decl. $=-09^{\circ} 43^{\prime} 05^{\prime \prime}$. 4 . The likely counterpart is Two Micron all Sky Survey (2MASS) 090913720943047 for which, apart from the magnitude (bmag $=20.0$ ), nothing else is known. The XMM-Newton spectrum is extremely hard. It can be well represented, in the $0.1-10 \mathrm{keV}$ energy range, by an absorbed power law with a photon index of $1.23_{-0.24}^{+0.33}$ and an absorption of $5.6_{-2.6}^{+5.4} \times 10^{21}$ atoms $\mathrm{cm}^{-2}$. The source flux extrapolated to the $10-40 \mathrm{keV}$ band is $(1.3 \pm 0.3) \times 10^{-12} \mathrm{erg}$ $\mathrm{cm}^{-2} \mathrm{~s}^{-1}$. It is thus clear that this single source accounts for the nonthermal flux detected both by Fusco-Femiano et al. (2003) and our analyses.

Valinia et al. (1999) and Fusco-Femiano et al. (2003) derived a lower limit for the magnetic field $B$ of $\sim 0.2 \mu \mathrm{G}$ and $\sim 0.1 \mu \mathrm{G}$, respectively. Our estimate of $B$, reported in Table 3, uses the Very Large Array (VLA) observations from Fusco-Femiano et al. (2003) $\left(S_{1365 \mathrm{MHz}}=86 \mathrm{mJy}, \alpha=1.5\right)$ and is consistent with the results of Bacchi et al. (2003) and of Fusco-Femiano et al. (2003).

\subsubsection{Coma}

Swift J1259.4+2757 is associated with the Coma cluster, which is one of the best studied clusters of galaxies. Coma (aka A1656) is a particularly rich and symmetric merging cluster. It has been known as a diffuse X-ray and radio source for 40 years (Felten et al. 1966; Forman et al. 1972; Willson 1970). The cluster hosts a powerful radio halo (Feretti \& Giovannini 1998), and both BeppoSAX (Fusco-Femiano et al. 1999) and RXTE (Rephaeli 2001; Rephaeli \& Gruber 2002) revealed the existence of nonthermal hard X-ray emission.

However, the detection of this hard X-ray excess is still quite controversial. Indeed, the positive BeppoSAX detections (Fusco-Femiano et al. 1999, 2004) of hard X-ray excess were challenged by Rossetti \& Molendi (2004) and Rossetti \& Molendi (2007). According to Rossetti \& Molendi (2007), the significance of the nonthermal excess changes (decreases) with the best-fit plasma temperature, and only a certain set of assumptions (e.g., temperature of the ICM) leads to a significant hard X-ray excess. However, recently, Fusco-Femiano et al. (2007), using different software analyses and studying a large set of background observations, were able to confirm their previous finding. Independent of the BeppoSAX results, the 

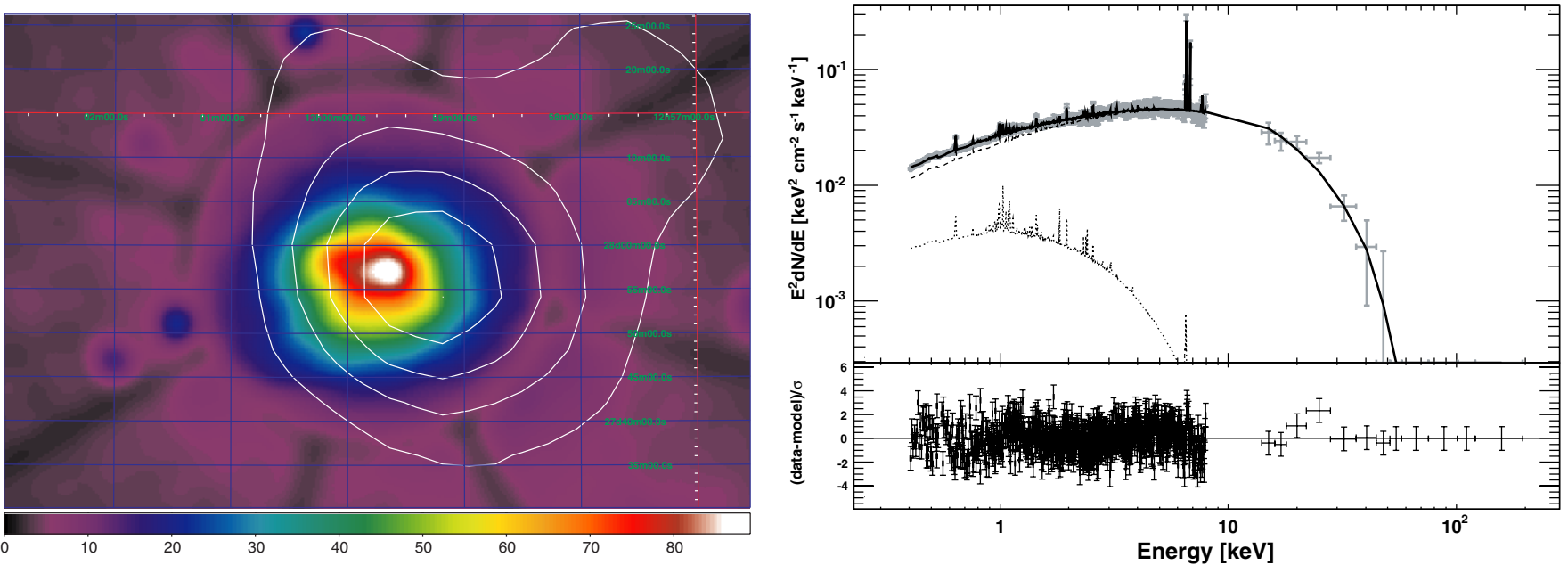

Figure 6. Left panel: ROSAT 0.1-2.4 keV surface brightness of Coma with BAT significance contours superimposed. The contours range from $2.5 \sigma$ to $20 \sigma$. Right panel: joint fit to XMM-Newton-BAT data. The best-fit model (solid line) is the sum of two thermal models (dashed and dotted lines).

(A color version of this figure is available in the online journal.)

RXTE detection (Rephaeli 2001; Rephaeli \& Gruber 2002) of the hard X-ray excess remains unchallenged.

Lately, Coma has also been targeted by INTEGRAL (Eckert et al. 2008a; Lutovinov et al. 2008). Eckert et al. (2008a) showed, in their combined XMM-Newton-INTEGRAL analysis, the presence of a hotter region (gas temperature of $12 \pm 2 \mathrm{keV}$ as compared to $7.9 \pm 0.1 \mathrm{keV}$ at the center) in the south-west region. The authors favored the possibility that this emission is produced by IC scattering because its spatial distribution overlaps the halo of radio synchrotron radiation. Lutovinov et al. (2008), by using INTEGRAL, ROSAT, and RXTE data, showed that the global Coma spectrum is well approximated only by a thermal emission model and found very marginal evidence $(1.6 \sigma)$ for hard X-ray excess. Thus, in light of these results, the evidence for nonthermal emissions in Coma does not seem conclusive.

Coma is the only cluster in our sample whose extent is larger than the BAT PSF. The analysis of point-like sources in the vicinity of the Coma cluster shows that the PSF full width at half-maximum (FWHM) is $22^{\prime}$ while the FWHM of the Coma detection is $26^{\prime}$. Using a simple Gaussian profile for the surface brightness of Coma yields a $1 \sigma$ extent in the $10^{\prime}-15^{\prime}$ range. This is in agreement with the morphological analysis of Eckert et al. (2008a). Moreover, from Figure 6, the offset between the BAT and the ROSAT centroids is apparent. Indeed, the BAT centroid falls $\sim 4^{\prime}$ west of the ROSAT surface-brightness peak. As discussed by Eckert et al. (2008a) and Lutovinov et al. (2008) for INTEGRAL, the high-energy centroid coincides with a region of hot gas likely due to an infalling subcluster.

Coded-mask detectors suppress the flux of diffuse sources and in order to recover the exact source flux and significance, one needs to develop dedicated methods for the analysis of extended objects (e.g., Renaud et al. 2006b). Given the fact that Coma is the only cluster "resolved" by BAT, a dedicated analysis will be left to a future paper (M. Ajello et al. 2008, in preparation). However, we can extract the spectrum treating Coma as a point-like source. This translates into an analysis of the source emission within a radius of $\sim 10^{\prime}$ from the BAT centroid. The BAT spectrum is well fitted by a thermal model with a gas temperature of $9.13_{-1.31}^{+1.68} \mathrm{keV}$.

XMM-Newton observed Coma several times. We analyzed an observation of $16 \mathrm{ks}$, which took place in 2005 June. The
XMM-Newton spectrum was extracted (as described in Section 2.2) including all photons within $10^{\prime}$ from the BAT centroid. Integrating the surface-brightness profile derived by ROSAT (beta model with $\beta=0.74$ and core radius $R_{c}=10.7$; Lutovinov et al. 2008) shows that our selection includes $\sim 75 \%$ of the total Coma flux. A fit to the XMM-Newton-BAT spectrum with a single-temperature model does not yield satisfactory results $\left(\chi^{2} /\right.$ dof $\left.=1168.9 / 858\right)$. We then tried to add a power law to the APEC model. Adding a power-law model improves the fit $\left(\chi^{2} /\right.$ dof $\left.=905.5 / 856\right)$ and results in a wellconstrained photon index of $2.11_{-0.13}^{+0.11}$. However, this fit leaves evident ("snake"-like) residuals at low energy (see below for the residuals of all Coma fits). These residuals might highlight the presence of another thermal component. Indeed, we find that a satisfactory fit $\left(\chi^{2} /\right.$ dof $\left.=846.5 / 856\right)$ is achieved using two APEC models. The most intense component has a temperature of $8.40_{-0.24}^{+0.25} \mathrm{keV}$ and an abundance of $0.21_{-0.03}^{+0.03}$, consistent with what was found by Arnaud et al. (2001) and Lutovinov et al. (2008). The low-temperature component $\left(T=1.45_{-0.11}^{+0.21} \mathrm{keV}\right.$ and $Z=0.05( \pm 0.02) Z_{\odot}$ ) very likely accounts for one or more of the X-ray sources in the field of Coma. Indeed, a hardness ratio analysis of these X-ray sources shows that their spectra are compatible with thermal models with temperatures in the $0.1-2 \mathrm{keV}$ range (Finoguenov et al. 2004). According to Finoguenov et al. (2004), these objects are (non-AGN) galaxies with a suppressed X-ray emission due to reduced starformation activity. Summarizing, we believe that the doublethermal model explains the data better than the thermal plus power-law model because (1) it produces the largest improvement in the fit (i.e., largest $\Delta \chi^{2}$ ), (2) it better reproduces the low-energy part of the spectrum, and (3) it accounts for all the point-like sources that are present in the XMM-Newton observation. The best fit, the sum of two APEC models, is shown in Figure 6. The residuals of all the fits described in this section are reported in Figure 7 while their parameters are summarized in Table 5.

Our $99 \% \mathrm{CL}$ upper limit in the $50-100 \mathrm{keV}$ band is $1.70 \times$ $10^{-12} \mathrm{erg} \mathrm{cm}^{-2} \mathrm{~s}^{-1}$. However, we remark that this spectrum is representative only of the $10^{\prime}$ radius region centered on the BAT centroid. Indeed, since the IC and the thermal emissions are proportional to the electron density and to its square, respectively $\left(F_{\mathrm{IC}} \propto n_{e}\right.$ and $F_{\text {thermal }} \propto n_{e}^{2}$; e.g., Sarazin et al. 

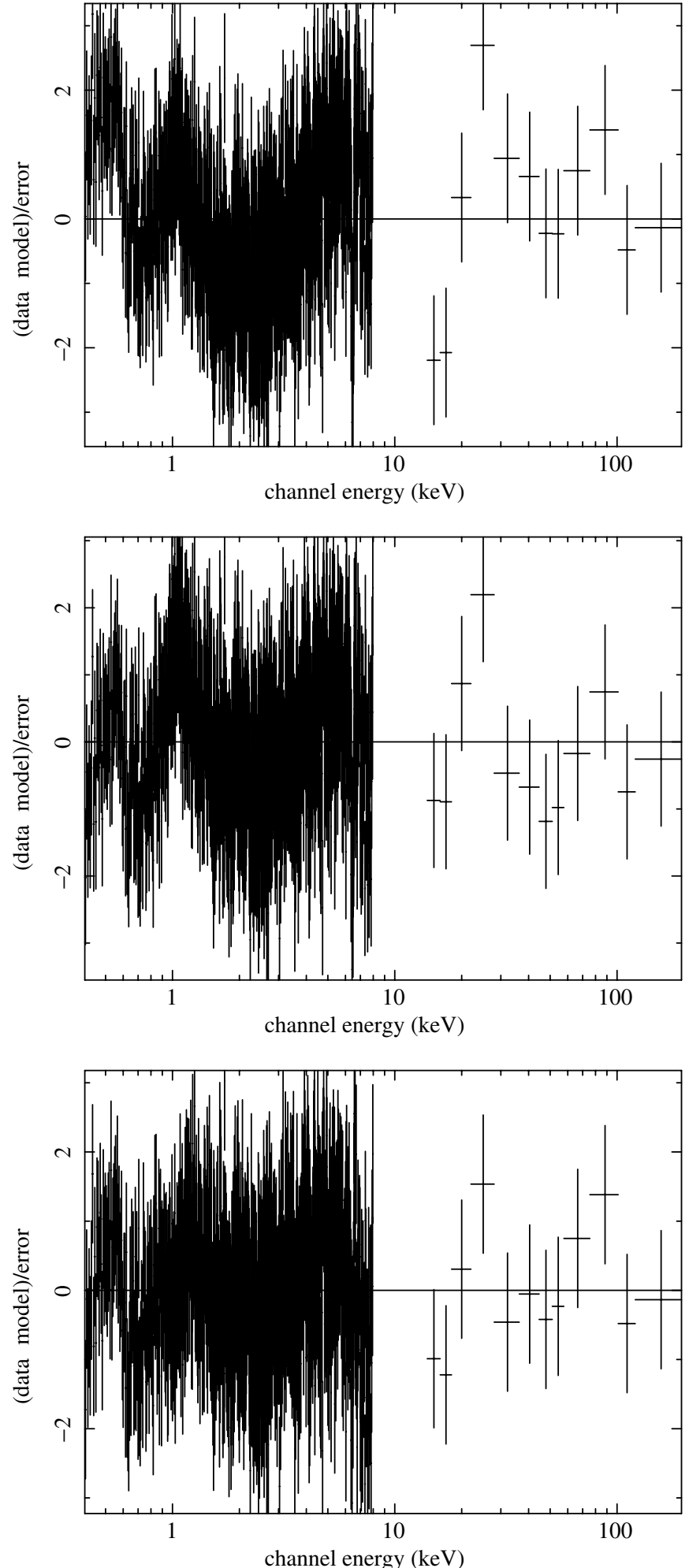

Figure 7. Residuals to the fit to Coma data using a single thermal model (top), sum of a thermal model and a power law (middle), and the sum of two thermal models (bottom).

1998a), their ratio (IC/thermal) is expected to increase with the distance from the cluster. Moreover, the lower density and larger sound speed (with respect to the physical conditions in the core) make CR acceleration more efficient in the outskirts of the cluster (Pfrommer et al. 2007). For these reasons and because Coma is an extended source for BAT, of which we
Table 5

Comparison of Different Spectral Fits to the Clusters Which Show a Large Deviation Between the ICM Temperature as Measured Below and Above $10 \mathrm{keV}$

\begin{tabular}{lccc}
\hline \hline Cluster & Thermal & Thermal + Power Law & Thermal + Thermal \\
\hline A2029 & & & \\
$k T_{1}$ & $6.75_{-0.31}^{+0.52}$ & $6.78_{-0.33}^{+0.46}$ & $4.1_{-1.5}^{+1.7}$ \\
$\Gamma$ & & 2.0 & \\
Norm. & & $1.55_{-1.15}^{+1.12} \times 10^{-3}$ & \\
$k T_{2}$ & & $9.6_{-2.0}^{+2.0}$ \\
$\chi^{2} /$ dof & $407.3 / 364$ & $402.1 / 363$ & $394.2 / 363$ \\
\hline Triangulum A & & & \\
$k T_{1}$ & $9.30_{-0.30}^{+0.30}$ & $9.25_{-0.28}^{+0.30}$ & $11.1_{-0.27}^{+0.34}$ \\
$\Gamma$ & & 2.0 & \\
Norm. & & $1.40 \times 10^{-4}$ & \\
$k T_{2}$ & & $1.63_{-0.27}^{+0.46}$ \\
$\chi^{2} /$ dof & $925.8 / 1074$ & $925.8 / 1073$ & $916.5 / 1072$ \\
\hline $\mathbf{A 2 3 1 9}$ & & & $11.2_{-1.0}^{+0.8}$ \\
$k T_{1}$ & $9.23_{-0.27}^{+0.27}$ & $9.33_{-0.52}^{+0.35}$ & \\
$\Gamma$ & & $1.7_{-0.3}^{+0.2}$ & \\
Norm. & & $7.8_{-5.3}^{+2.7} \times 10^{-4}$ & $1.9_{-0.40}^{+1.64}$ \\
$k T_{2}$ & & $1139.9 / 1272$ & $1127.8 / 1272$ \\
$\chi^{2} /$ dof & $1151.34 / 1274$ & & \\
Coma & & & \\
$k T_{1}$ & $6.50_{-0.05}^{+0.09}$ & $7.19_{-0.06}^{+0.16}$ & $8.40_{-0.24}^{+0.25}$ \\
$\Gamma$ & & $2.11_{-0.13}^{+0.10}$ & \\
Norm & & $3.56_{-0.34}^{+0.46} \times 10^{-3}$ & $1.45_{-0.11}^{+0.21}$ \\
$k T_{2}$ & & $905.5 / 856$ & $846.5 / 856$ \\
$\chi^{2} /$ dof & $1168.9 / 858$ & & \\
\hline
\end{tabular}

Notes. As a reference for the reader, the parameters of Coma are also reported. $k T_{1}$ and $k T_{2}$ are the temperatures of the two thermal models (in $\mathrm{keV}$ ) while norm. and $\Gamma$ are the normalization at $1 \mathrm{keV}$ (in $\mathrm{ph}^{-2} \mathrm{~s}^{-1}$ $\mathrm{keV}^{-1}$ ) and the photon index of the power-law model, respectively. Frozen parameters do not have an error estimate.

analyze only the core, we cannot exclude the presence of a nonthermal component that arises in the outskirts of the cluster.

$$
\text { 2.4.6. } A 3571
$$

Swift J1347.7 - 3253 is likely associated with the A3571 cluster, which has also been detected in the RXTE Slew-Survey (Revnivtsev et al. 2004). Its symmetric morphology (see the left panel of Figure 8) and temperature map indicate that A3571 is a relaxed cluster (e.g., Markevitch et al. 1998). However, the radio structure, of the complex in which A3571 lies, suggests that this cluster is in the late stages of merging (Venturi et al. 2002). We note that A3571 is known to have a moderately cool core (Peres et al. 1998). Past and recent studies do not report evidence for nonthermal hard X-ray emission in A3571. A fit to the BAT spectrum with a bremsstrahlung model yields a temperature of $6.9_{-2.6}^{+6.0} \mathrm{keV}$ (in agreement with the mean temperature of $6.71_{-0.42}^{+0.15} \mathrm{keV}$ measured with Chandra by Sanderson et al. 2006), but the chi-square $\left(\chi_{\text {red }}^{2}=1.76\right)$ is relatively poor. The BAT spectrum shows positive residuals above $60 \mathrm{keV}$, which might reveal the presence of a hard tail (see Figure 8). However, given the low $\mathrm{S} / \mathrm{N}$ of our spectrum, adding a power-law component does not improve the chi-square. XMM-Newton observed A3571 for $12 \mathrm{ks}$ in 2007 July. According to Nevalainen et al. (2001), the surface brightness of A3571 follows a beta profile with $\beta=0.68$ and core radius $R_{c}=3 .^{\prime} 85$. Therefore, our region of $10^{\prime}$ radius includes approximately $93 \%$ of the cluster emission. This factor is taken into account when performing the joint fit 

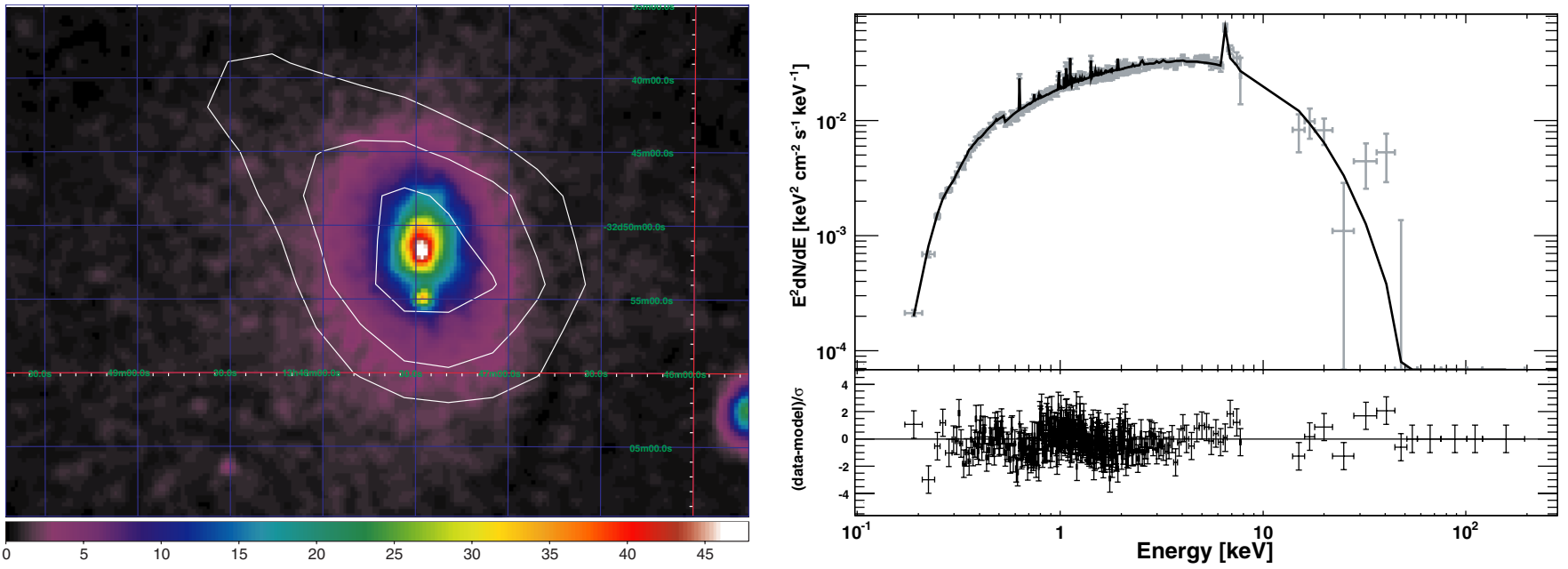

Figure 8. Left panel: ROSAT 0.1-2.4 keV surface brightness of A3571 with BAT significance contours superimposed. The contours range from $2.5 \sigma$ to $5.0 \sigma$. Right panel: joint fit to XMM-Newton-BAT data with a thermal model. The best-fit model is shown as a solid line.

(A color version of this figure is available in the online journal.)

of XMM-Newton and BAT data. The combined XMM-NewtonBAT spectrum, shown in the right panel of Figure 8, is well fitted by an APEC model with a plasma temperature of $6.01 \pm 0.21$ $\mathrm{keV}$ and an abundance of $0.34 \pm 0.06$ solar. The total $2-10 \mathrm{keV}$ flux of $(8.0 \pm 0.3) \times 10^{-11} \mathrm{erg} \mathrm{cm}^{-2} \mathrm{~s}^{-1}$ is in good agreement with the value of $(7.3 \pm 0.4) \times 10^{-11} \mathrm{erg} \mathrm{cm}^{-2} \mathrm{~s}^{-1}$ measured by BeppoSAX (Nevalainen et al. 2001). Even though statistically not required, a nonthermal power law (photon index fixed to 2.0) is well constrained by our data. Indeed, we are able to derive a $50-100 \mathrm{keV}$ flux of $(1.4 \pm 0.5) \times 10^{-12} \mathrm{erg} \mathrm{cm}^{-2} \mathrm{~s}^{-1}$.

The radio flux density from the NRAO ${ }^{9}$ VLA Sky Survey is $S_{1380 \mathrm{MHz}}=8.4 \mathrm{mJy}$ (Condon et al. 1998). We could not find any reference for the spectral index, so we adopted the value of $\alpha=1.5$, which leads to the lower limit listed in Table 3 . We note that a steeper spectrum gives a larger upper limit for the magnetic field (e.g., $\alpha=2$ ) and would yield a lower limit twice as large as the previous one.

\subsubsection{A2029}

Swift J1511.0+0544 is likely associated with the A2029 cluster, which has also been detected at high energy by RXTE, BeppoSAX, and Chandra (Revnivtsev et al. 2004; Molendi \& De Grandi 1999; Clarke et al. 2004, respectively). The left panel of Figure 9 shows that the BAT source is well centered on the cluster emission as seen by ROSAT. A2029 has a moderately cool core (Sarazin et al. 1998b; Molendi \& De Grandi 1999). Clarke et al. (2004) presented an analysis of Chandra observations of the central region and found signs of interactions between the X-ray and the radio plasma. The unusual central radio source (PKS0745-191) morphology would be typical of a merging cluster. They suggested that A2029 is a cluster that very recently started to cool to lower temperatures.

The BAT data alone are well fitted $\left(\chi^{2} /\right.$ dof $\left.=6.89 / 10\right)$ by a simple bremsstrahlung model with a temperature of $10.6_{-3.3}^{+5.8} \mathrm{keV}$. An $8 \mathrm{ks}$ long XRT observation took place in 2005 September. Given the extent of the XRT CCD, we extracted all the photons within $6^{\prime}$ from the BAT centroid. The surfacebrightness profile follows a beta model with $\beta=0.64$ and core

\footnotetext{
9 The National Radio Astronomy Observatory is a facility of the Nationa Science Foundation operated under cooperative agreement by Associated Universities, Inc.
}

radius $R_{c}=1^{\prime} .8$ (Sarazin et al. 1998a). Integrating the beta profile up to $6^{\prime}$ yields that $95 \%$ of the total cluster emission is included in our selection. However, for the case of A2029, the beta profile fails to explain the inner $1^{\prime} 8$ region, which is characterized by a bright core (Sarazin et al. 1998a). Thus, our selection might include a higher fraction of the total cluster emission. Indeed, BAT and XRT data are well fitted without the need for a cross-normalization constant. The BAT and XRT data are successfully fitted by an APEC model with a plasma temperature of $7.45 \pm 0.34 \mathrm{keV}$ and a solar abundance of $0.39 \pm 0.09$, which is consistent with the Chandra results (Clarke et al. 2004). From the combined fit, we derive a $99 \%$ CL upper limit to the nonthermal flux in the $50-100 \mathrm{keV}$ band of $1.27 \times 10^{-12} \mathrm{erg} \mathrm{cm}^{-2} \mathrm{~s}^{-1}$. However, we note that the fit leaves positive residuals at high energy. We thus used a second APEC model, with abundance fixed at 0.4 , to account for them. The $f$-test confirms that the second thermal component is detected at $99.85 \%$ CL. The best-fit temperatures are $9.6_{-2.0}^{+2.0} \mathrm{keV}$ and $4.1_{-1.5}^{+1.7} \mathrm{keV}$, respectively. This fit is shown in Figure 9. A2029 has been targeted by ground-based TeV telescopes; however, no $\mathrm{TeV}$ emission has been detected so far (Perkins et al. 2006).

Condon et al. (1998) found $S_{1380 \mathrm{MHz}}=527.8 \mathrm{mJy}$. We adopted the value of $\alpha=1.5$, which leads to the lower limits on the magnetic field estimated in Table 3. We note that Taylor et al. (1994) obtained a lower limit on the magnetic field of $0.18 \mu \mathrm{G}$ using observations of the central radio galaxy.

$$
\text { 2.4.8. } A 2142
$$

Swift J1558.5+2714 is associated with the A2142 merging cluster. The detection in the $3-20 \mathrm{keV}$ band by $R X T E$ (Revnivtsev et al. 2004) makes the association of the BAT source with the cluster rather strong. According to Peres et al. (1998) and De Grandi \& Molendi (2002), A2142 has a cool core that survived the merger. Markevitch et al. (2000) and Sanderson et al. (2006), using Chandra observations, noted that the core of A2142 has a complex structure, probably with a poor cluster enclosed in the halo of a hotter larger cluster. This would explain the lower temperature in the center, without the presence of a cool core. The left panel of Figure 10 shows a point-like source located less than $4^{\prime}$ from the cluster center. This object is 

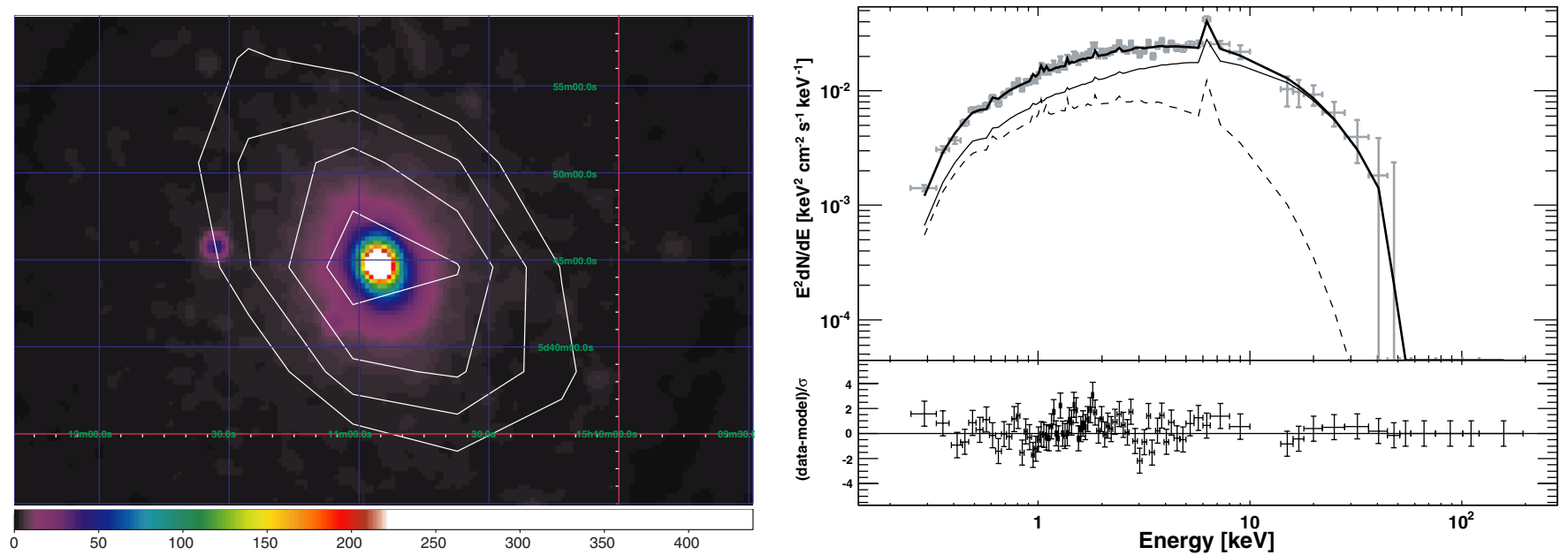

Figure 9. Left panel: ROSAT 0.1-2.4 keV surface brightness of A2029 with BAT significance contours superimposed. The contours range from $2.5 \sigma$ to $5.0 \sigma$. Right panel: joint XRT-BAT spectrum of A2029. The best fit (thick solid line) is the sum of two thermal models (thin solid and dashed line).

(A color version of this figure is available in the online journal.)
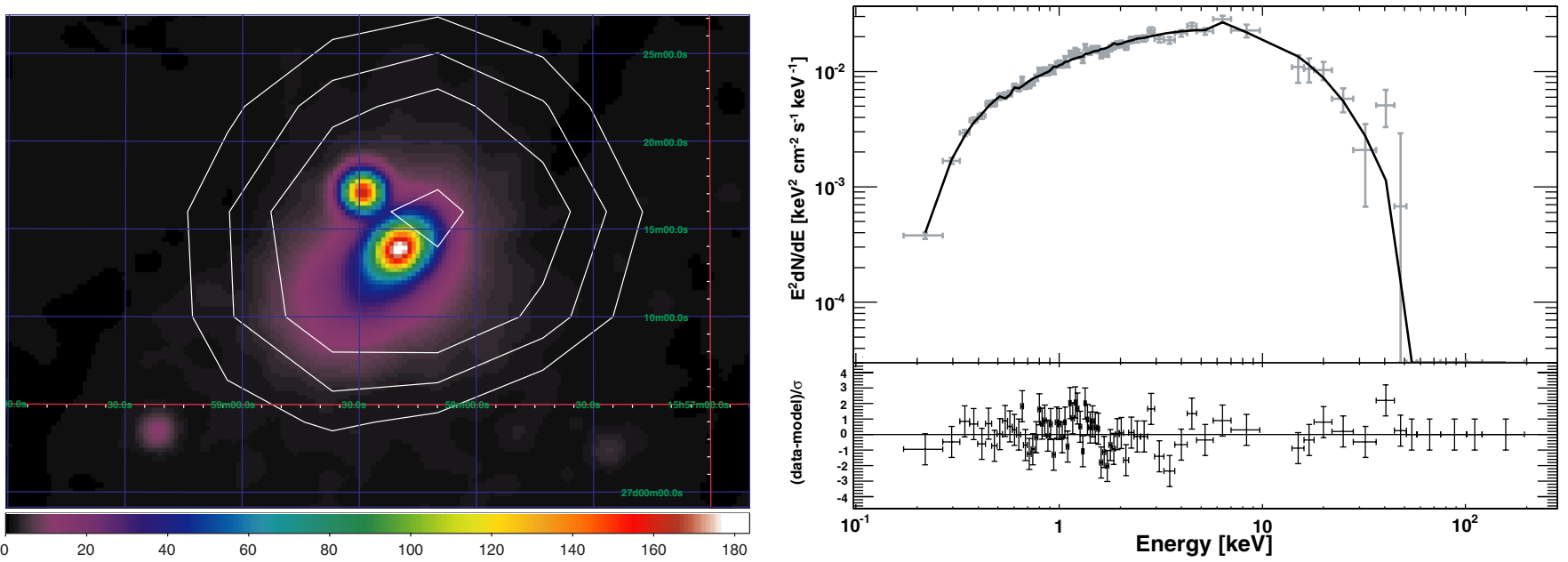

Figure 10. Left panel: ROSAT 0.1-2.4 keV surface brightness of A2142 with BAT significance contours superimposed. The contours range from $2.5 \sigma$ to $7.0 \sigma$. Right panel: joint fit to XMM-Newton-BAT data for A2142 with a single thermal model. The best-fit model is shown as a solid line.

(A color version of this figure is available in the online journal.)

the Seyfert 1 galaxy 2E 1556.4+2725. Given the distance, both objects, the cluster and the Sy1, are not separated by BAT.

The BAT data are well fitted by a simple bremsstrahlung model $\left(\chi^{2} /\right.$ dof $\left.=7.96 / 10\right)$ with a plasma temperature of $10.1_{-2.7}^{+3.7} \mathrm{keV}$. We analyzed an XMM-Newton observation of $800 \mathrm{~s}$ in conjunction with the BAT data. In this case, we separately extracted the spectrum of the cluster and the spectrum of the Sy1 2 E $1556.4+2725$. The latter shows an X-ray spectrum typical of a Sy1 object, that is, absorption consistent with the Galactic one and a photon index of $1.98_{-0.14}^{+0.16}$. The extrapolated flux in the $15-55 \mathrm{keV}$ range is $2.3 \times 10^{-12} \mathrm{erg} \mathrm{cm}^{-2} \mathrm{~s}^{1}$ and it is well below the BAT sensitivity. Therefore, we can consider the Sy1 contribution to be negligible in the BAT band. The surface brightness profile of A2142 follows a beta model with $\beta=0.83$ and core radius $R_{c}=4^{\prime} 2$ (Henry \& Briel 1996). Integrating the beta profile up to $10^{\prime}$ yields that $97 \%$ of the total cluster emission is included in our selection. However, for the case of A2142, the beta profile underestimates the brightness of the inner $3^{\prime}$ region, which is characterized by a bright core (Henry \& Briel 1996). Thus, our selection might include a higher fraction of the total cluster emission. Indeed, BAT and XMM data are well fitted without the need for a cross-normalization constant. The cluster XMM-Newton-BAT spectrum is well fitted by a simple APEC model with a plasma temperature of $8.40_{-0.45}^{+0.64} \mathrm{keV}$. The fit is shown in the right panel of Figure 10. This is in good agreement with the temperatures of $8.8_{-0.9}^{+1.2} \mathrm{keV}$ and $9.0 \pm$ $0.3 \mathrm{keV}$ measured by Chandra and Ginga, respectively (Markevitch et al. 2000; White et al. 1994). From our fit, the abundance is $0.27_{-0.13}^{+0.13}$ solar. Since no hard X-ray excess is detected, we report $99 \%$ CL upper limits. Using a power law with a photon index of 2.0, we derive from the XMM-Newton-BAT data a $99 \% \mathrm{CL}$ upper limit to the $50-100 \mathrm{keV}$ nonthermal flux of $1.6 \times 10^{-12} \mathrm{erg} \mathrm{cm}^{-2} \mathrm{~s}^{-1}$. The $99 \% \mathrm{CL}$ limit on the nonthermal luminosity is $6.1 \times 10^{43} \mathrm{erg} \mathrm{s}^{-1}$. The marginal $(\sim 2 \sigma)$ BeppoSAX detection of a nonthermal emission (Nevalainen et al. 2004 ) is a factor of 5 larger than our upper limit and is, thus, incompatible with our data.

The presence of a radio halo was already reported by Harris et al. (1977). Giovannini \& Feretti (2000) measured $S_{1400 \mathrm{MHz}}=$ $18.3 \mathrm{mJy}$. In the absence of a measured index $\alpha$, we adopt the arbitrary value of $\alpha=1.5$ to obtain the magnetic field constraint listed in Table 3. 

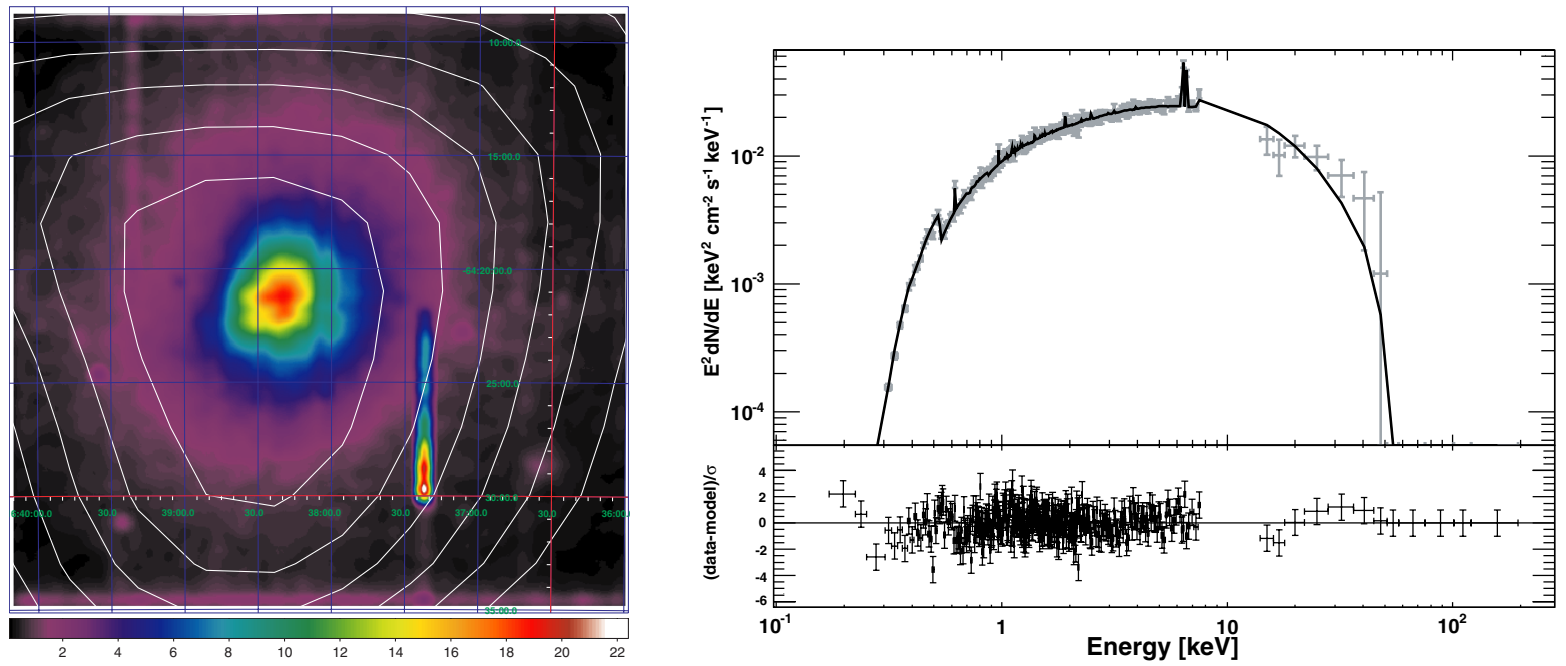

Figure 11. Left panel: XMM-Newton 1.0-7.0 keV surface brightness of the Triangulum Australis cluster with BAT significance contours superimposed. The contours range from $2.5 \sigma$ to $7.0 \sigma$. Right panel: joint fit to XMM-Newton-BAT data for the Triangulum Australis cluster with a thermal model. The best-fit model is shown as a solid line.

(A color version of this figure is available in the online journal.)

\subsubsection{Triangulum Australis}

Swift J1638.8 - 6424, shown in the right panel of Figure 11, is likely associated with the hot X-ray cluster of galaxies Triangulum Australis. This cluster at $z=0.058$ has already been detected in the ROSAT, RXTE Slew, and INTEGRAL surveys (Voges et al. 1999; Revnivtsev et al. 2004; Stephen et al. 2006). In particular, the detections by RXTE and INTEGRAL above $15 \mathrm{keV}$ make this association certain. The Triangulum Australis cluster may host a cool core (Edge et al. 1992; Peres et al. 1998). However, Markevitch et al. (1996) used the temperature and entropy maps from ASCA and ROSAT to find an indication of the probable presence of a subcluster merger, and argued that the cool gas in the core does not require a cooling flow. Markevitch et al. (1998) found that a nonthermal component is more likely than a cooling flow.

The BAT spectrum is well fitted $\left(\chi^{2} /\right.$ dof $\left.=5.68 / 9\right)$ by a simple bremsstrahlung model with a plasma temperature of $13.4_{-3.7}^{+6.3} \mathrm{keV}$. A similar temperature was found by Markevitch et al. (1996) in the center of the cluster.

XMM-Newton observed the Triangulum Australis cluster for $7480 \mathrm{~s}$ in 2001 February. According to the beta profile reported by Markevitch et al. (1996), selecting photons within $10^{\prime}$ of the BAT centroid includes $\sim 92 \%$ of the cluster emission. We thus employ such a cross-normalization factor when fitting $X M M-N e w t o n$ and BAT data. The BAT and XMM-Newton data are consistent with a pure APEC model. From the best fit, shown in the right panel of Figure 11, we derive a plasma temperature of $9.30_{-0.30}^{+0.30} \mathrm{keV}$ and an abundance of $0.30_{-0.07}^{+0.07}$ solar. The $X M M-N e w t o n-\mathrm{BAT}$ temperature is in agreement with the mean values of $9.06_{-0.31}^{+0.33} \mathrm{keV}$ and $9.50 \pm 0.70 \mathrm{keV}$, reported by Ikebe et al. (2002) and Chen et al. (2007), respectively. Using a power law with a photon index of 2.0, we derive a $99 \%$ CL upper limit to nonthermal emission in the $50-100 \mathrm{keV}$ band of $6.5 \times$ $10^{-13} \mathrm{erg} \mathrm{cm}^{-2} \mathrm{~s}^{-1}$.

Condon et al. (1993) reported a $4.85 \mathrm{GHz}$ radio source centered $\sim 7^{\prime}$ away from the BAT centroid. They found an upper limit of $33 \mathrm{Jy}$. We adopt this flux and the arbitrary value of $\alpha=1.5$ to obtain the magnetic field constraint listed in Table 3 .

\subsubsection{Ophiucus}

Swift J1712.3 - 2319 lies only 1'.7 (see Figure 12) away from one of the most studied galaxy clusters, Ophiucus, discovered by Johnston et al. (1981). The detection at high energies by BeppoSAX and INTEGRAL (Nevalainen et al. 2001; Bird et al. 2006 , respectively) makes the association with the BAT source certain. Watanabe et al. (2001a) used ASCA to measure the $\mathrm{X}$-ray brightness distribution and temperature map. Considering the similarities with the Coma cluster, they concluded that Ophiucus is not relaxed and has likely experienced a recent merger. The BAT-derived plasma temperature of $9.5_{-1.1}^{+1.4} \mathrm{keV}$ is in good agreement with the values of $9.6_{-0.5}^{+0.6} \mathrm{keV}$ and $9.0_{-0.3}^{+0.3}$ $\mathrm{keV}$ measured by BeppoSAX (Nevalainen et al. 2001) and by Suzaku (Fujita et al. 2008).

A hard X-ray excess was detected by Nevalainen et al. (2001) at a $2 \sigma$ level. Very recently, Eckert et al. (2008b), using INTEGRAL, confirmed this hard X-ray emission at a higher

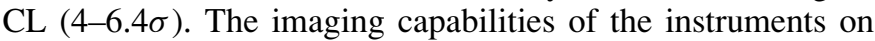
board INTEGRAL allowed the authors to conclude that the observed excess over the thermal emission does not originate from point sources (such as obscured AGNs) and is therefore nonthermal. This excess is marginally consistent with BAT data. Indeed, from our data, we derive a $90 \%$ upper limit to the nonthermal component $(20-60 \mathrm{keV})$ of $7.2 \times 10^{-12} \mathrm{erg} \mathrm{cm}^{-2}$ $\mathrm{s}^{-1}$ while the reported nonthermal flux observed by INTEGRAL is $(10.1 \pm 2.5) \times 10^{-12} \mathrm{erg} \mathrm{cm}^{-2} \mathrm{~s}^{-1}$.

We analyzed an archival Chandra observation of $\sim 50 \mathrm{ks}$. The observation, which took place in 2002 October, was performed using the Advanced CCD Imaging Spectrometer (ACIS-S). Given its extent, the Ophiucus cluster is not entirely contained in a single chip. We thus extracted only those photons in a region of radius of $2^{\prime} .1$ around the BAT centroid. The region extent is dictated by the size of the chip. When performing a simultaneous fit with BAT data, we must, therefore, account for the flux that falls outside of the ACIS-S chip. Assuming that the surface density follows a beta profile and adopting the values of $\beta=0.64$ and core radius of $R_{c}=3.2$ as found by Watanabe et al. (2001b) and confirmed by Eckert et al. (2008b), we derive that only $\sim 52 \%$ of the total cluster flux 

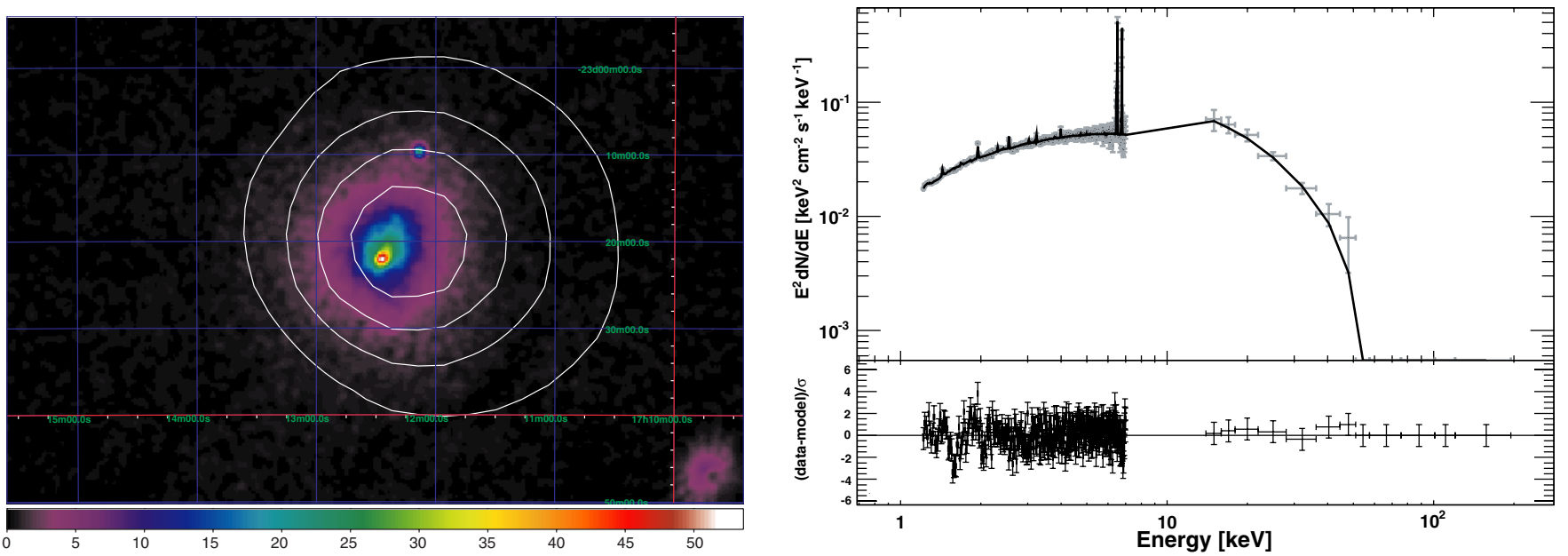

Figure 12. Left panel: ROSAT 0.1-2.4 keV surface brightness of the Ophiucus cluster with BAT significance contours superimposed. The contours range from $2.5 \sigma$ to $22 \sigma$. Right panel: joint fit to Chandra-BAT data for the Ophiucus cluster with a thermal model. The best fit is shown as a solid line.

(A color version of this figure is available in the online journal.)

is included in our selection. If we let the cross-normalization of the BAT and the Chandra data vary, we derive that the Chandra data show a normalization (with respect to the BAT ones) of $53_{-6}^{+5} \%$, which is in good agreement with the $52 \%$ derived above. Thus, we fix the cross-normalization factor at $52 \%$. Moreover, as in Blanton et al. (2003), we account for the uncertainty in the background subtraction by adding a systematic uncertainty of $2 \%$. The joint Chandra-BAT spectrum is well fitted by a single APEC model with a temperature of $9.93_{-0.24}^{+0.24} \mathrm{keV}$ and an abundance of $0.52 \pm 0.03$. Using a power law with a photon index of 2.0, we derive a 99\% CL upper limit to the nonthermal emission in the $50-100 \mathrm{keV}$ and $20-60 \mathrm{keV}$ bands of $2.8 \times$ $10^{-12} \mathrm{erg} \mathrm{cm}^{-2} \mathrm{~s}^{-1}$ and $4.5 \times 10^{-12} \mathrm{erg} \mathrm{cm}^{-2} \mathrm{~s}^{-1}$, respectively. The INTEGRAL detection is inconsistent $(\sim 2 \sigma)$ with our upper limit.

The Ophiucus cluster is associated in the radio domain with the extended radio source MSH 17-203 (Johnston et al. 1981). The most recent radio data date back to 1977 (Slee 1977) and report $S_{160 \mathrm{MHz}}=6.4 \mathrm{Jy}$ and $\alpha=2$, which we use to produce the lower limit on the magnetic field reported in Table 3. The results do not change if we use older radio measurements (e.g., Mills et al. 1960; Jones \& Finlay 1974; Slee \& Higgins 1975).

\subsubsection{A2319}

Swift J1920.9+4357 is certainly associated with the massive A2319 cluster, which undergoes a major merger (e.g., O'Hara et al. 2004). The BAT centroid (see the left panel of Figure 13) lies $\sim 2^{\prime}$ north-west of the peak of the ROSAT emission. Indeed, Chandra observations reveal at the same position a region of hot $(\sim 12 \mathrm{keV})$ gas while at the position of the ROSAT peak there is likely a cool core (O'Hara et al. 2004). A2319 has been detected above $10 \mathrm{keV}$ by BeppoSAX and RXTE (Molendi et al. 1999; Gruber \& Rephaeli 2002, respectively). These two measurements are symptomatic of the uncertainty related to the hard X-ray detection claims from nonimaging instruments and the inherent uncertainty from source contamination. Indeed, Molendi \& De Grandi (1999) reported that no hard-tail emission is present in BeppoSAX data, while Gruber \& Rephaeli (2002) found that a power-law component can explain some residual features in the $15-30 \mathrm{keV}$ energy range. The BAT data favor the thermal scenario. Indeed, the best fit to the data is obtained using a pure bremsstrahlung model with a plasma temperature of $14.1_{-3.0}^{+4.0} \mathrm{keV}$ consistent, within the large errors, with the $9.6 \pm 0.3 \mathrm{keV}$ value measured by BeppoSAX.

In addition, we analyzed a $10 \mathrm{ks} X M M$-Newton observation together with the BAT data. Utilizing the surface-brightness profile obtained by O'Hara et al. (2004) (beta model with $\beta=0.55$ and core radius $R_{c}=2.6^{\prime}$ ), we determine that our region of $10^{\prime}$ radius includes $\sim 90 \%$ of the cluster emission. We employ such cross-normalization factor when fitting $X M M-N e w t o n$ and BAT data. The BAT-XMM-Newton spectra, shown in the right panel of Figure 13, are well fitted by an APEC model with a plasma temperature of $9.27_{-0.27}^{+0.27} \mathrm{keV}$ and an abundance of $0.25( \pm 0.04)$ solar. The $99 \%$ upper limit on the $2-10 \mathrm{keV}$ nonthermal flux of $2.70 \times 10^{-12} \mathrm{erg} \mathrm{cm}^{-2} \mathrm{~s}^{-1}$ is in disagreement with the nonthermal flux of $(4.0 \pm 0.1) \times 10^{-11}$ erg $\mathrm{cm}^{-2} \mathrm{~s}^{-1}$, detected in the same band by RXTE (Gruber \& Rephaeli 2002).

Harris \& Miley (1978) discovered a diffuse radio halo associated with the A2319 cluster. An intensive study was done by Feretti et al. (1997), from which we take $S_{610 \mathrm{MHz}}=1 \mathrm{Jy}$ and $\alpha=0.92$ to estimate the lower limit of the magnetic field reported in Table 3.

\section{CLUSTERS PROPERTIES}

\subsection{Constraints on Nonthermal Excess Emission}

In order to constrain the nonthermal hard X-ray emission, we have produced $3 \sigma$ upper limits on the $50-100 \mathrm{keV}$ nonthermal flux for each source presented in the previous section. We excluded the Perseus and the Coma clusters. Indeed, Perseus is the only cluster where the detected "hard-tail" is certainly produced by the brightest AGN while Coma requires a dedicated analysis. We chose the 50-100 keV energy band because the thermal emission of the clusters is negligible above $50 \mathrm{keV}$.

The $3 \sigma$ upper limit has been computed by integrating the source flux in the $50-100 \mathrm{keV}$ range and subtracting the thermal flux arising from the best thermal fit. We added to this value three times the $1 \sigma$ uncertainty. The upper limits are reported in Table 3. These upper limits were derived using BAT data alone. It is important to note that, indeed, thanks to the very good sensitivity of BAT, all these upper limits are very stringent. Indeed, the nonthermal flux for all these sources is constrained to be below $\sim 1 \mathrm{mCrab}$. 

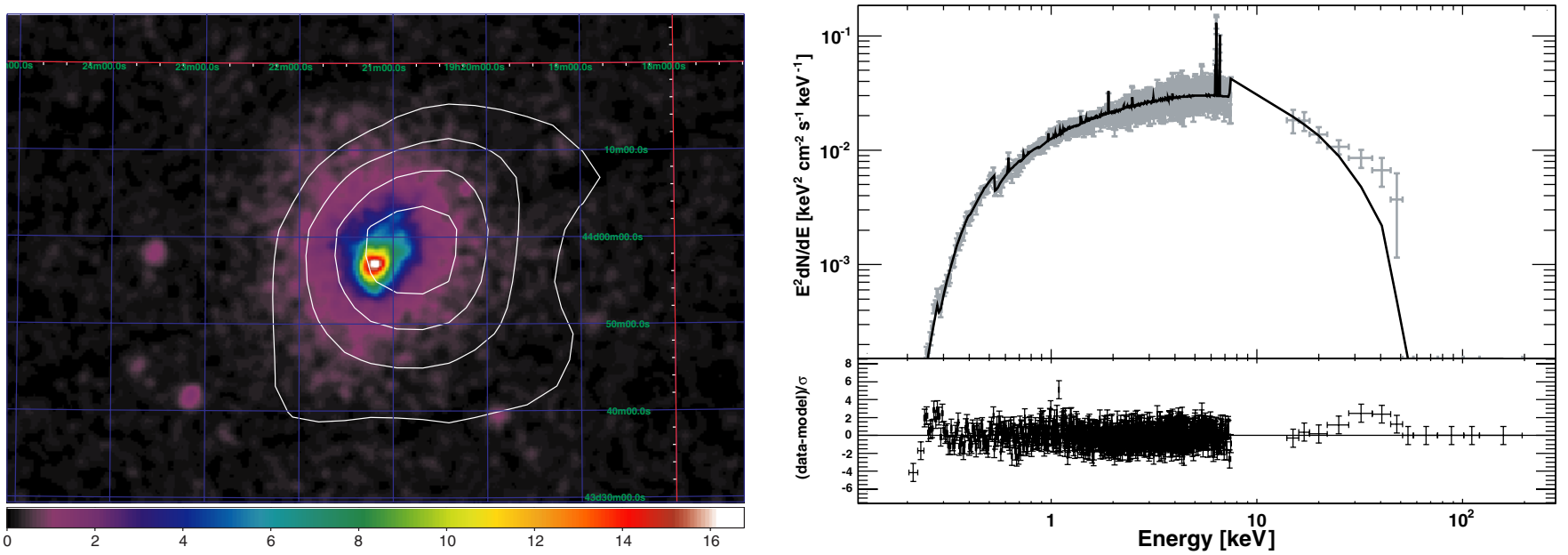

Figure 13. Left panel: ROSAT 0.1-2.4 keV surface brightness of A2319 with BAT significance contours superimposed. The contours range from $2.5 \sigma$ to $22 \sigma$. Right panel: joint fit to XMM-Newton-BAT data for A2319 Australis cluster. The best-fit model thermal model is shown as a solid line. (A color version of this figure is available in the online journal.)

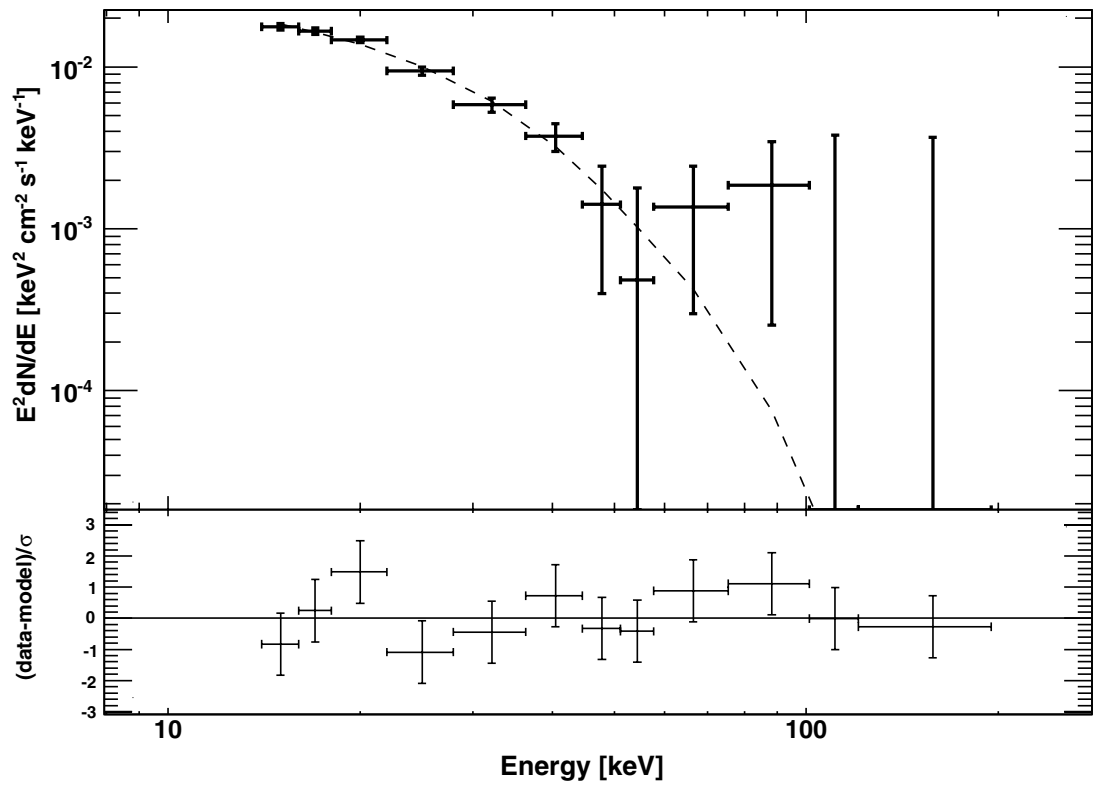

Figure 14. Stacked spectrum of the clusters in our sample and the best fit (dashed line) with a bremsstrahlung model.

In the above derivation, we do not make any assumption on the mechanism generating the nonthermal flux. However, in most cases, IC scattering is believed to be the principal emission process (e.g., Sarazin 1999; Nevalainen et al. 2004; Fusco-Femiano et al. 2007; Eckert et al. 2008b). If this is true, then the IC emission can be modeled as a power law with a photon index of $\sim 2$ in the 1-200 keV energy range (see, e.g., Reimer et al. 2004). We thus computed the $99 \%$ CL upper limits to the IC flux in the $50-100 \mathrm{keV}$ band by adding a power-law model to the best fits reported in Table 2. These limits are reported in Table 4. It is worth noting that, since we are using $X M M-N e w t o n / X R T / C h a n d r a$ and BAT data, these upper limits are a factor of 5-10 lower than those derived using BAT data alone (see Table 3 ).

\subsection{Stacking Analysis}

A few clusters show positive, marginal, residuals above $50 \mathrm{keV}$; this is the case for A3266, A3571, and A2142. Such features are not statistically significant to warrant an additional component (e.g., nonthermal power law). However, it might be that the nonthermal component is just below the BAT sensitivity for such clusters. In this case, the stacking technique offers the capability to explore the average properties of a given population beyond the current instrumental limit. Thus, we produced the stacked spectrum of all clusters except Perseus and Coma (for the reasons explained above). The average spectrum is produced by the weighted average of all the spectra. The weight is chosen to be the inverse of the variance of a given bin and is exactly the same procedure as that used to extract the spectra of each individual source. The same stacking technique has been applied with success to the study of Seyfert galaxies detected by BAT (Ajello et al. 2008c). The total spectrum has an exposure time of $\sim 56 \mathrm{Ms}$, and it is shown in Figure 14. A fit with a simple bremsstrahlung model yields a good chi-square $\left(\chi^{2} / \mathrm{dof}=7.2\right.$ / 10). The best-fit temperature is $10.8_{-0.8}^{+0.9} \mathrm{keV}$, which is in very good agreement with the mean temperature of $10.4 \mathrm{keV}$ as 


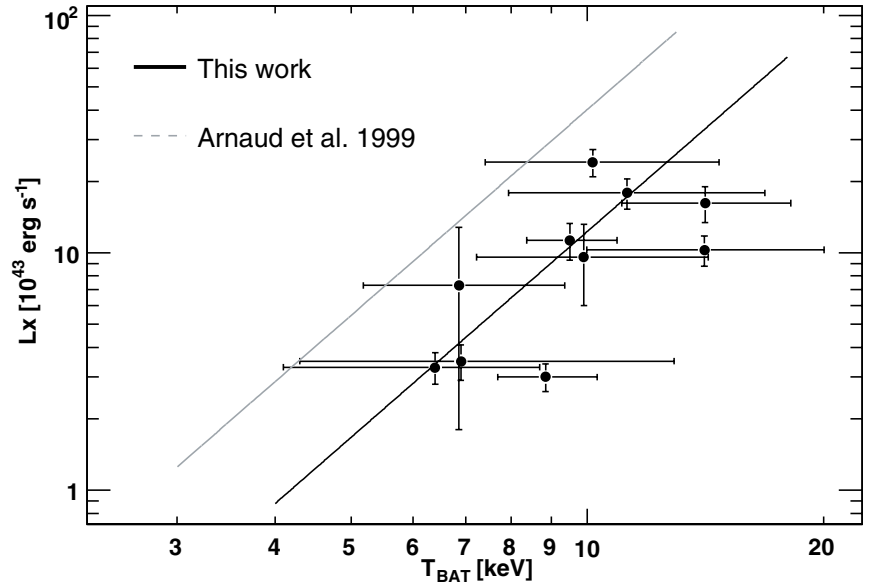

Figure 15. Luminosity-temperature relation for the BAT clusters. The black line is the best, power-law, fit to the data while the gray line is the best fit of Arnaud \& Evrard (1999) converted to the BAT energy band.

determined by averaging the values obtained by fitting a simple bremsstrahlung model to each cluster's spectrum (using BAT data alone). This is a good confirmation that the chosen stacking technique well reproduces the average properties of our cluster sample.

From the best thermal fit, we derive a 99\% CL upper limit (50-100 keV) for the nonthermal component of $1.9 \times$ $10^{-12} \mathrm{erg} \mathrm{cm}^{-2} \mathrm{~s}^{-1}(0.3 \mathrm{mCrab})$. At the average redshift of the sample $(z=0.058)$, this translates into a limiting luminosity of $1.4 \times 10^{43} \mathrm{erg} \mathrm{s}^{-1}$. Nevalainen et al. (2004) reported the detection of an average nonthermal component detected in the stacked spectrum (20-80 keV) of BeppoSAX clusters. Their nonthermal luminosity is ${ }^{10}$ in the $(0.5-5.0) \times 10^{43} \mathrm{erg} \mathrm{s}^{-1}$ range. In the 20 $80 \mathrm{keV}$ band, our $99 \%$ CL limit on the nonthermal luminosity is $2.2 \times 10^{43} \mathrm{erg} \mathrm{s}^{-1}$. Thus, the findings of Nevalainen et al. (2004) are consistent with our analysis.

On the other hand, all clusters, except perhaps Perseus and A3571, undergo a merging phase. These last two clusters are those that show the lowest ICM temperatures in our sample. The $L_{x}-T$ relation (shown in Figure 15) reinforces the picture that most of the BAT clusters are mergers. Indeed, the best fit to the data with a power law of the form $L=A_{6} T_{6}^{\alpha}$ where $T_{6}=T / 6 \mathrm{keV}$ (fixing $\alpha$ at $2.88^{11}$ ) yields a normalization $A_{6}=(2.82 \pm 0.8) \times 10^{43} h_{70}^{-2} \mathrm{erg} \mathrm{s}^{-1}$, whereas Markevitch (1998) and Arnaud \& Evrard (1999) found for $A_{6}$ the values of $(12.53 \pm 1.08) \times 10^{43} h_{70}^{-2} \mathrm{erg} \mathrm{s}^{-1}$ and $(12.13 \pm 0.06) \times 10^{43} h_{70}^{-2}$ erg $\mathrm{s}^{-1}$, respectively. Indeed, merging clusters are known to segregate at lower luminosities (or higher temperatures) in the $L_{x}-T$ plane (Ota et al. 2006).

There is growing evidence that points toward a rather nonuniform distribution of temperatures in the ICM of merging clusters (e.g., Markevitch et al. 2003; O'Hara et al. 2004; Eckert et al. 2008a). Both hydrodynamical simulations (e.g., Takizawa 1999) and observations (see Markevitch et al. 2003, for A0754) have shown that shocks due to cluster mergers can heat the ICM up to $\sim 15 \mathrm{keV}$. Figure 16 shows that, for the merging clusters, the mean temperature measured by BAT is slightly higher (given the large uncertainties) than the mean ICM temperature

\footnotetext{
10 The measurement reported by Nevalainen et al. (2004) had to be converted to the Hubble constant used in this paper.

11 Given the small range in luminosity spanned by our sample, we fixed $\alpha$ at the value determined by Arnaud \& Evrard (1999).
}

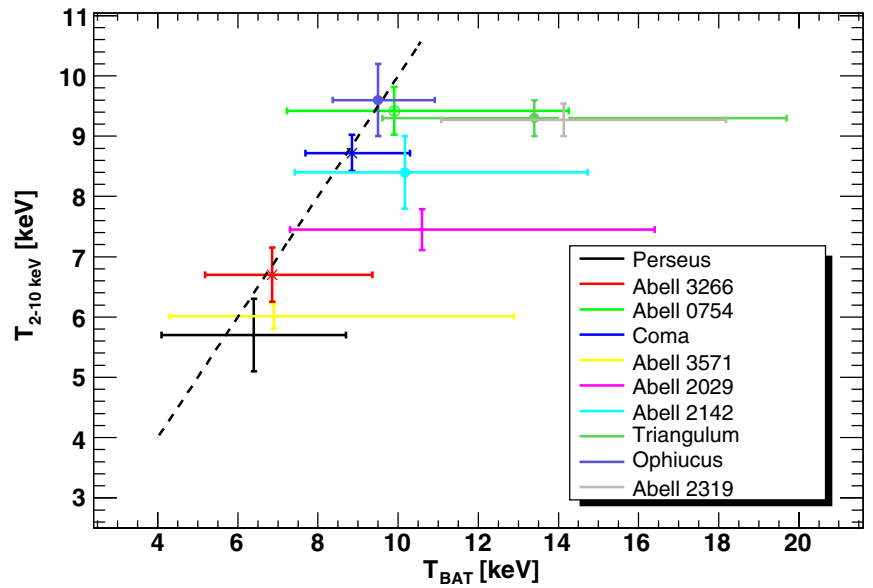

Figure 16. Comparison of best-fit gas temperatures. The $x$-axis reports the temperatures derived using BAT data (above $15 \mathrm{keV}$ ) while the $y$-axis shows the temperatures derived using 2-10 keV data (XMM-Newton, Chandra, or XRT). The dashed line shows the $T_{\mathrm{BAT}}=T_{2-10 \mathrm{keV}}$ function. The largest deviations are for the merging clusters A2029 and A2319.

measured below $10 \mathrm{keV}$. A similar trend, although using different wavebands, has been recently reported for a sample of 192 galaxy clusters (Cavagnolo et al. 2008). Moreover, for the merging clusters, the BAT centroid is shifted to positions where Chandra and XMM-Newton have detected regions of hot gas. Based on this evidence, we believe that the conjecture that these clusters show regions of "hot" gas is a more viable claim than the one that foresees the presence of a strong IC component.

This claim is also supported by the fact that the high-energy residuals (e.g., residuals above $10 \mathrm{keV}$ of the spectral fits using a single thermal model) are, in general, better described by an additional thermal component than a power-law model. To prove this, we selected those clusters that show, in the analysis presented in Section 2.2, the largest residuals above $10 \mathrm{keV}$ from the thermal model used. These clusters, which are A2029, Triangulum Australis, and A2319, also show a large deviation between the ICM temperature measured below and above $10 \mathrm{keV}$ (see Figure 16). We made a fit to each of these clusters with (1) a single thermal model, (2) the sum of a thermal and a power-law model, and (3) the sum of two thermal models. The residuals to each of these fits are shown in Figures 17-19 while the spectral parameters are summarized in Table 5. We note that, in all three cases, the additional thermal model explains the residuals better than an additional power-law model. We also remark that, for most of the BAT clusters (in this case for Triangulum Australis and A2319), the single thermal model is already a good description of the data $\left(\chi^{2} /\right.$ dof $\left.=\sim 1.0\right)$, and, given the statistics, no other additional model is required. This means that, currently, the high-energy residuals (with respect to a single thermal fit) are not significant. Longer BAT exposures will clarify the existence and nature of these emissions.

\subsection{Cluster Magnetic Field Assessment}

The diffuse synchrotron radio emission (radio halos, relics, and mini-halos) proves the existence of magnetic fields in the ICM. The intensity of the synchrotron emission depends on both the strength of the magnetic field and the electron density. If the nonthermal X-ray emission results from IC scattering of the same radio electrons by the $\mathrm{CMB}$, then the degeneracy in magnetic field and relativistic electron density can 

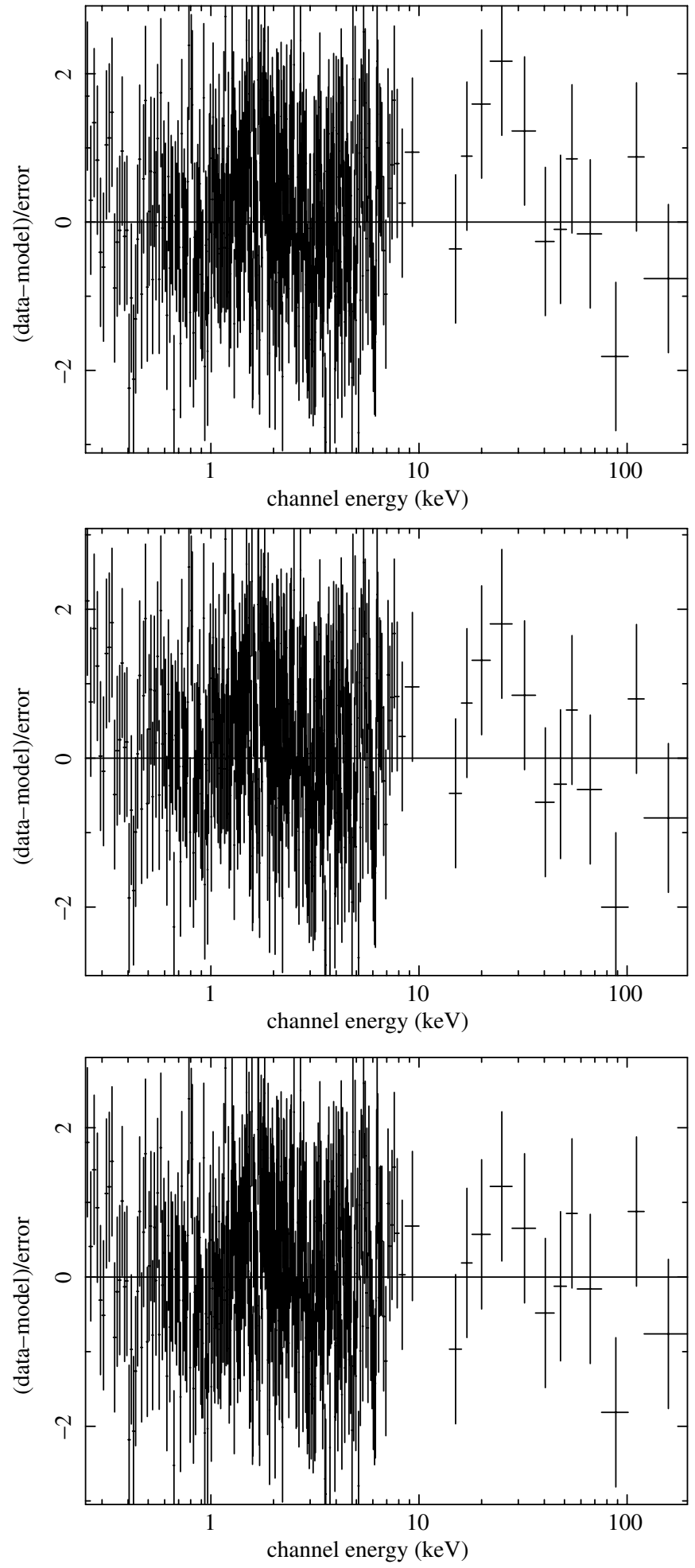

Figure 17. Residuals of the fit to A2029 data using a single thermal model (top), sum of a thermal model and a power law (middle), and the sum of two thermal models (bottom).

be broken (e.g., Rephaeli 2001). Therefore, the nondetection of a nonthermal component can be used to place a lower limit on the magnetic fields $B$ in the clusters (the ratio of IC to radio flux is inversely proportional to $\left.B^{\alpha+1}\right)$. Following Harris \& Romanishin (1974) and Sarazin (1988), we estimate the lower limit on $B$ (the
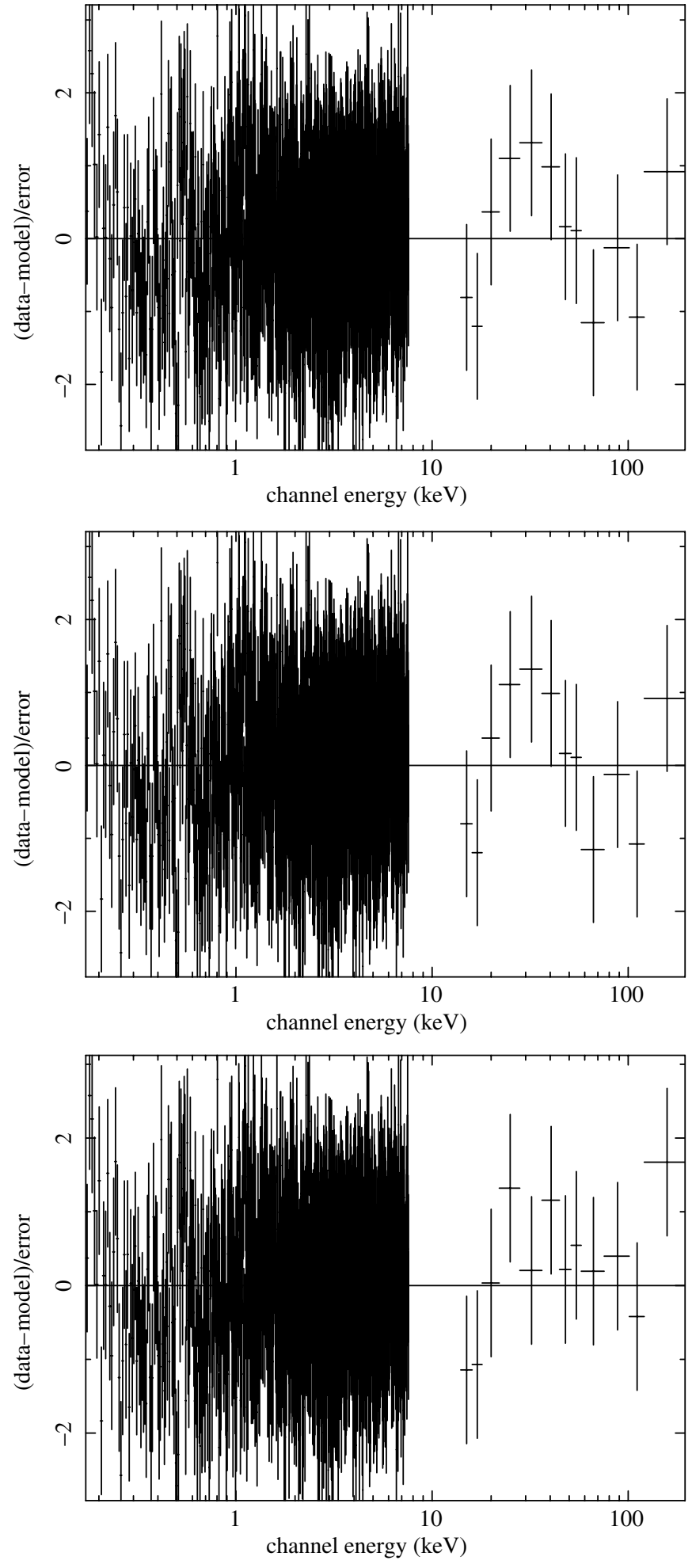

Figure 18. Residuals of the fit to Triangulum Australis data using a single thermal model (top), sum of a thermal model and a power law (middle), and the sum of two thermal models (bottom).

volume-averaged component along the line of sight):

$\frac{f_{x} \nu_{r}^{-\alpha}}{s_{r}\left(\int_{\nu_{\min }}^{v_{\max }} v_{x}^{-\alpha} d \nu_{x}\right)}=\frac{2.47 \times 10^{-19} T_{C M B}^{3} b(p)}{B a(p)}\left(\frac{4960 T_{C M B}}{B}\right)^{\alpha}$,

where $\alpha$ is the spectral index, $p=2 \alpha+1, f_{x}$ is the $\mathrm{X}$-ray flux integrated over the band between $v_{\min }=50 \mathrm{keV}$ and $v_{\max }=$ $100 \mathrm{keV}\left(f_{x}=k_{c} \int_{v_{\min }}^{v_{\max }} v_{x}^{-\alpha} d v_{x}\right.$, in erg $\left.\mathrm{cm}^{-2} \mathrm{~s}^{-1}\right), s_{r}=k_{s} v_{r}^{-\alpha}$ 

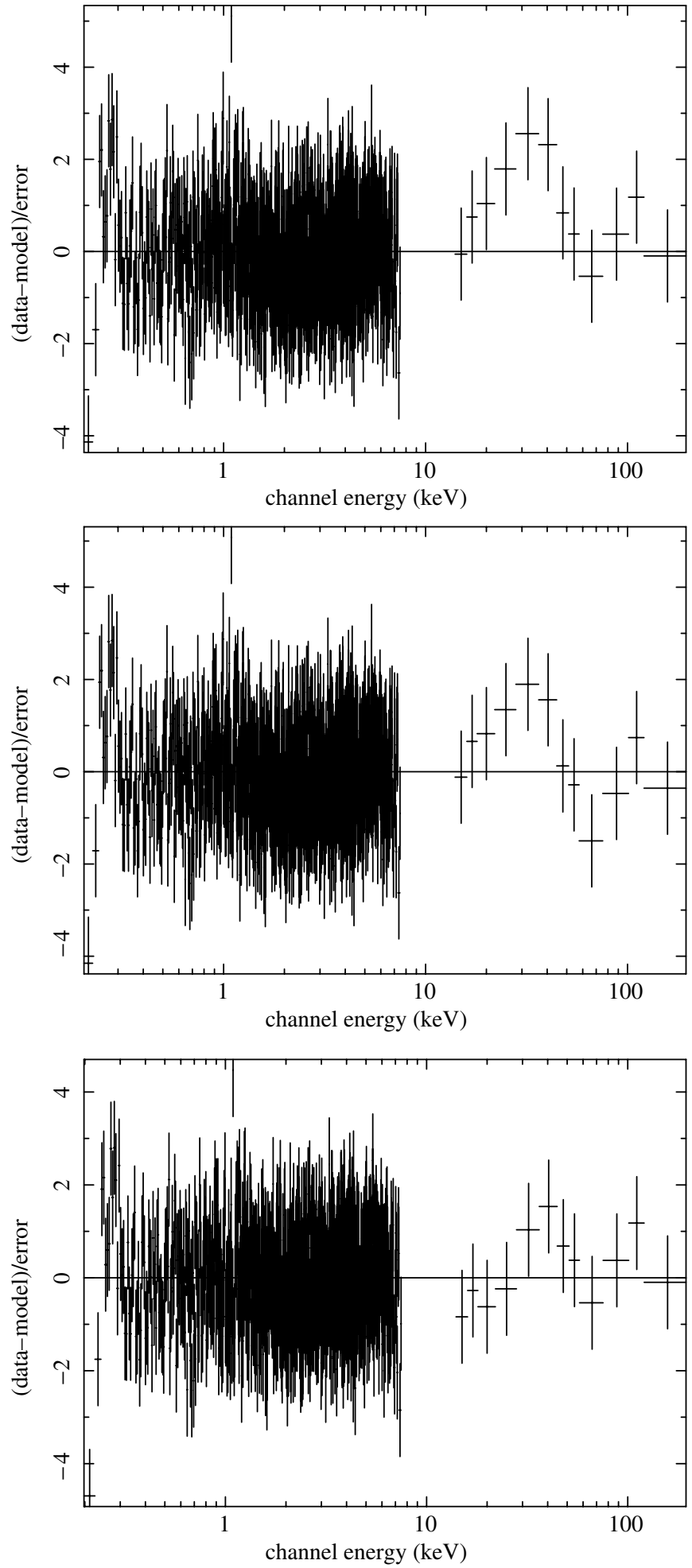

Figure 19. Residuals of the fit to A2319 data using a single thermal model (top) sum of a thermal model and a power law (middle), and the sum of two thermal models (bottom).

the flux density at the radio frequency $v_{r}$ (in erg $\mathrm{cm}^{-2} \mathrm{~s}^{-1} \mathrm{~Hz}^{-1}$ ), $T_{\mathrm{CMB}}=2.7 \mathrm{~K}$ the temperature of the $\mathrm{CMB}$, and $a(p)$ and $b(p)$ as in Sarazin (1988; Equations 5.6 and 5.8). Since our clusters are nearby, in the above formula we neglect redshift corrections.

Although the limit on the X-ray flux is very stringent, the measurement of the diffuse radio emission is complicated by the presence of individual radio galaxies in the cluster. In most cases, the radio observations were not sensitive enough over

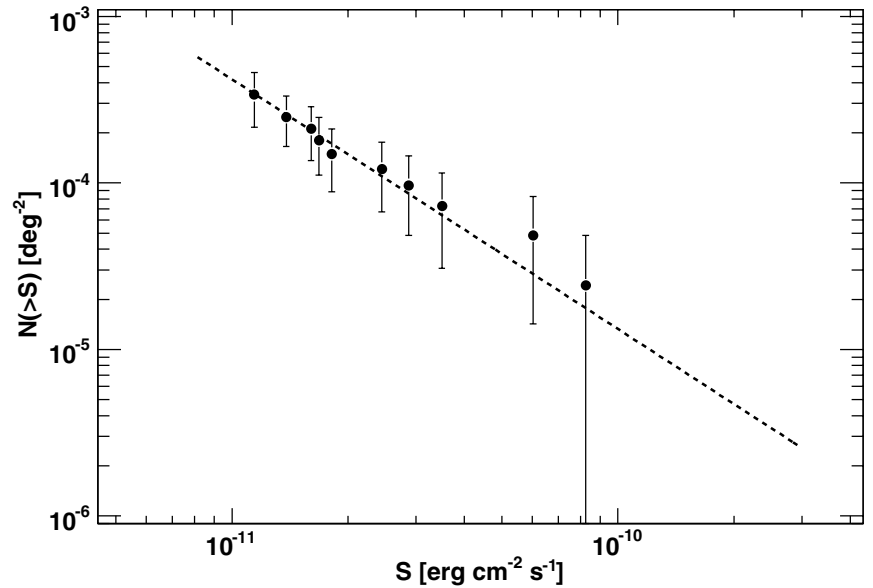

Figure 20. Cumulative flux number relation for the BAT clusters (15-55 keV). The dashed line is an overlaid power law $\mathrm{N}(>\mathrm{S})=\mathrm{A} \mathrm{S}^{-1.5}$.

a wide range of spatial scales to subtract the contribution of the single sources. Moreover, the spectral index varies with the distance from the center of the cluster. These factors make the derivation of the magnetic field intensity uncertain. Therefore, the values listed in Table 3 have to be taken as order-ofmagnitude estimates. Such estimates point to magnetic fields that are typically a fraction of a $\mu \mathrm{G}$. These low values indicate that these systems are far from equipartition. This is possible if one considers that the magnetic fields and the relativistic particles may have a different spatial extension and history.

The magnetic field can also be evaluated by measuring the Faraday rotation measure (RM) of the plane of polarization from the radio galaxies in the cluster or in the background (e.g., Kim et al. 1991; Clarke et al. 2001). The two estimates are different (with $B_{\mathrm{RM}} \gg B$ ), most likely because the interpretation of Faraday rotation measurements and the derivation of the mean magnetic field strength rests on assumptions of the magnetic field topology (see Goldshmidt \& Rephaeli 1993; Colafrancesco et al. 2005, for an extensive discussion). We can produce a more robust upper limit of the IC flux considering that the IC emission spectrum can be approximated as a power law in the $1-200 \mathrm{keV}$ energy band (see, e.g., Reimer et al. 2004, for more details). Using both $2-10 \mathrm{keV}$ and BAT data, we are able to produce the limits reported in Table 4, which are, in some cases, a factor of 5-10 lower than our previous estimated values (Table 3) based on BAT data alone. This, in turn, translates into larger intensities of the magnetic field which, in a few cases, reach the $\sim 0.5 \mu \mathrm{G}$ value.

\section{CLUSTER POPULATION}

\subsection{Cluster $\log N-\log S$}

Thanks to the serendipitous character of the BAT survey, it is possible to derive, for the first time, the source counts distribution (also known as $\log N-\log S$ ) of clusters above $15 \mathrm{keV}$. This can be obtained as

$$
N(>S)=\sum_{i=0}^{N_{S}} \frac{1}{\Omega_{i}}\left(\operatorname{deg}^{-2}\right),
$$

where $N_{S}$ is the total number of clusters with fluxes greater than $S$ and $\Omega_{i}$ is the geometrical area surveyed with that limiting flux. The cumulative distribution is reported in Figure 20. Source count distributions are generally fitted by a power law of the 
form $N(>S)=A S^{-\alpha}$. Given the small number of objects, we do not attempt a maximum likelihood fit to derive the slope $\alpha$, but we note that our flux distribution is consistent with an Euclidean function $N \propto S^{-3 / 2}$, as shown in Figure 20. We derive the normalization $A$ as the one that reproduces the number of observed objects above the flux of $\sim 1 \times 10^{-11} \mathrm{erg} \mathrm{cm}^{-2}$ $\mathrm{s}^{-1}$. Using the $90 \%$ CLs for small numbers derived by Gehrels (1986), we find that a good representation of our data is obtained by $N(>S)=\left(4.19_{-1.4}^{+2.1} \times 10^{-4} \mathrm{deg}^{-2}\right) S_{11}^{-1.5}$, where $S_{11}$ is the flux in units of $10^{-11} \mathrm{erg} \mathrm{cm}^{-2} \mathrm{~s}^{-1}$. This function is also shown in Figure 20.

Interestingly, we note that the integrated flux of all clusters above $10^{-11} \mathrm{erg} \mathrm{cm}^{-2} \mathrm{~s}^{-1}$ is $9.7 \times 10^{-11} \mathrm{erg} \mathrm{cm}^{-2} \mathrm{~s}^{-1} \mathrm{sr}^{-1}$. This is only $\sim 0.1 \%$ of the Cosmic X-ray Background (CXB) flux as measured by BAT in the 15-55 keV band (Ajello et al. 2008b), but $5-10 \%$ of the total flux resolved by BAT into AGNs (Ajello et al. 2008c). Thus, clusters of galaxies are a sizeable population among the extragalactic objects (mostly AGNs) detected by BAT.

We can compare the BAT $\log N-\log S$ with those derived in the $0.5-2 \mathrm{keV}$ band. In doing so, we extrapolate the BAT spectra to the $0.5-2 \mathrm{keV}$ band using the temperatures measured below $10 \mathrm{keV}$. The cluster surface density above $10^{-12} \mathrm{erg} \mathrm{cm}^{-2} \mathrm{~s}^{-1}$ in the $0.5-2.0 \mathrm{keV}$ band is $4.3_{-2.3}^{+3.0} \times 10^{-2} \mathrm{deg}^{-2}$, which is in rather good agreement with the findings of Vikhlinin et al. (1998) and Burenin et al. (2007).

The BAT source counts distribution can be used to estimate the foreseen number of galaxy clusters above a given flux limit. In doing so, we adopt for $\alpha$ the -1.4 value, which has been established by deeper X-ray surveys (e.g., Jones et al. 1998; Böhringer et al. 2001, and references therein). Indeed, using the $-3 / 2$ value would certainly overestimate the cluster density at lower fluxes. As an example, an instrument surveying the whole sky to $10^{-13} \mathrm{erg} \mathrm{cm}^{-2} \mathrm{~s}^{-1}$ would detect approximately $\sim 10,000$ galaxy clusters in the $15-55 \mathrm{keV}$ band. The BAT sample itself will comprise of up to 30 objects, if BAT will able to reach the 0.5 mCrab flux limit on the whole sky.

\subsection{X-Ray Luminosity Function}

Since all our clusters have a measured redshift, we can derive their luminosity function. Its construction relies on the knowledge of the survey volume $V_{\max }$ as a function of X-ray luminosity. The survey volume is the volume of the cone defined by the survey area and the luminosity distance at which a cluster with a given luminosity could just be observed at the flux limit. The limiting luminosity distance $D_{L \text { lim }}$, and thus also $V_{\max }$, can be determined by iteratively solving the following equation:

$$
D_{L \lim }^{2}=\frac{L_{x}}{4 \pi F_{\lim } k(T, z)},
$$

where $L_{x}$ is the source luminosity and $k(T, z)$ is the $k$-correction, which accounts for the redshifting of the source spectrum.

Once the $V_{\max }$ is computed for each object, the cumulative luminosity function can be derived as

$$
N\left(>L_{x}\right)=\sum_{i=0}^{N} \frac{1}{V_{\max }\left(L_{i}\right)}\left(h_{70}^{3} \mathrm{Mpc}^{-3}\right) .
$$

The cumulative luminosity function of the BAT clusters, obtained with the method reported above, is shown in Figure 21. Böhringer et al. (2002), analyzing a flux-limited sample

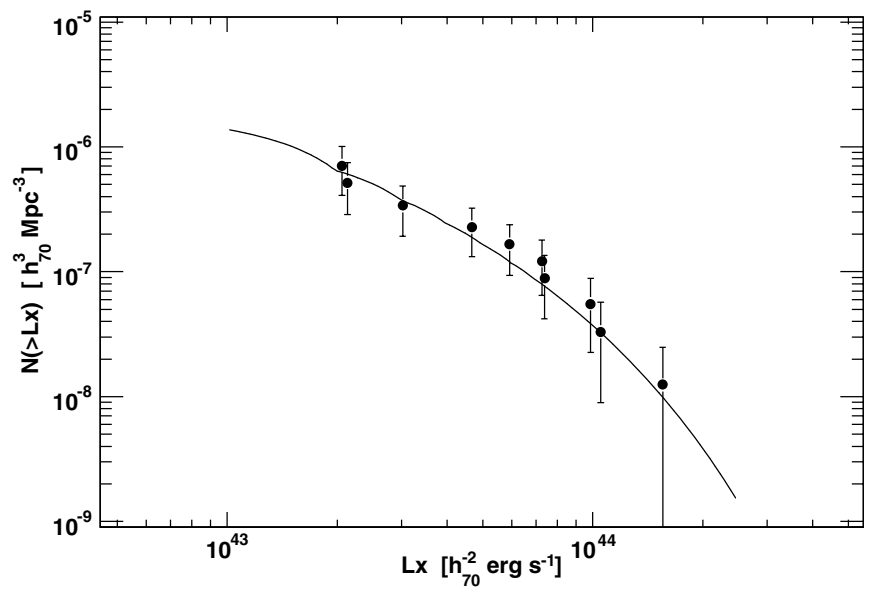

Figure 21. Cumulative luminosity function of the BAT clusters (15-55 keV). The solid line is the X-ray luminosity function determined for the REFLEX survey (Böhringer et al. 2002) converted to the BAT energy band.

of ROSAT galaxy clusters (REFLEX), derived that a good parametrization of the differential luminosity function is a Schechter function of the form

$$
\frac{d N}{d L}=n_{0} \exp \left(-\frac{L}{L_{*}}\right)\left(\frac{L}{L_{*}}\right)^{-\alpha} \frac{1}{L_{*}} .
$$

In order to compare the REFLEX luminosity function with that of the BAT, we adopt for $n_{0}, L_{*}$, and $\alpha$ the values determined by Böhringer et al. (2002). Moreover, since the REFLEX luminosity function is derived in the $0.1-2.4 \mathrm{keV}$ band, we need to convert the luminosities to the BAT $15-55 \mathrm{keV}$ band. We do this by using the mean clusters temperature $(k T=8.1 \mathrm{keV})$, determined in the $2-10 \mathrm{keV}$ band (see the right panel of Figure 16). The reason for adopting this temperature instead of the BAT-derived temperature is twofold. First, given the $\mathrm{S} / \mathrm{N}$, temperatures determined in the $2-10 \mathrm{keV}$ band have a better accuracy than temperatures determined in the BAT band. Second and most importantly, however, using the $2-10 \mathrm{keV}$ temperature allows a more accurate extrapolation of the source luminosity from the ROSAT $(0.1-2.4 \mathrm{keV})$ to the BAT $(15-55 \mathrm{keV})$ band. The extrapolated, cumulative, REFLEX luminosity function is also reported in Figure 21. It is apparent that, notwithstanding the extrapolation, the agreement of the BAT data and the REFLEX luminosity function is excellent. This agreement is not, however, surprising because most of the BAT clusters constitute the bright end of the REFLEX luminosity function. The value of $L_{*}$ converted to the $15-55 \mathrm{keV}$ band is $L_{*}=7.3 \times 10^{43} h_{70}^{-1} \mathrm{erg} \mathrm{s}^{-1}$ while $n_{0}=5.13_{-1.8}^{+2.7} \times 10^{-7}$ and $\alpha=1.63$.

Integrating the luminosity function multiplied by the luminosity yields the total X-ray emissivity $W$ of galaxy clusters. Above the survey limit of $2 \times 10^{43} \mathrm{erg} \mathrm{s}^{-1}$, we find ${ }^{12} W=2.83 \times$ $10^{37} \mathrm{erg} \mathrm{s}^{-1} \mathrm{Mpc}^{-3}$ (15-55 keV). This can be compared to the total emissivity of AGN, which was derived for the local Universe, and a similar energy band (17-60 keV) by Sazonov et al. (2007). After correcting for the small difference between the energy bands, the AGN local emissivity above $2 \times 10^{43} \mathrm{erg} \mathrm{s}^{-1}$ is $W_{\mathrm{AGN}}=14.1 \times 10^{37} \mathrm{erg} \mathrm{s}^{-1} \mathrm{Mpc}^{-3}$. It is thus clear that galaxy clusters substantially contribute $(\sim 20 \%$ level with respect to AGN) to the local X-ray output.

12 We do not provide an error estimate since the luminosity function was not fitted to the data. 


\section{DISCUSSION}

\subsection{Nonthermal Hard X-Ray Emission}

Direct evidence of the presence of relativistic electrons in the ICM arises from the existence of large radio halos (Dennison 1980; Feretti \& Giovannini 2007). The same electron population responsible for the synchrotron emission can, in principle, scatter CMB photons by IC and produce hard Xray radiation. The intensity of this radiation relative to the synchrotron emission ultimately depends on the value of the magnetic field.

A firm detection of nonthermal components in the spectra of galaxy clusters has remained elusive in the past as well as in this study. Indeed, Perseus is the only galaxy cluster in the BAT sample where a nonthermal high-energy component is revealed at high significance. Most likely, this component is due to the emission of the central AGN NGC 1275. The rest of the clusters detected by BAT do not show a significant nonthermal emission. Using BAT data alone, we are able to constrain the nonthermal component below the $\mathrm{mCrab}$ level in the 50-100 keV energy band. The BeppoSAX detection above $50 \mathrm{keV}$ of an average nonthermal component in the stacked spectrum of several clusters is consistent with the BAT upper limit (Nevalainen et al. 2004). As discussed in Section 2.2, some of the individual detections of nonthermal components (e.g., Eckert et al. 2008b) are consistent (albeit some marginally) with the upper limits derived using BAT data alone. Thus, we cannot exclude that such nonthermal components exist and that they are currently below or at the limit of the BAT sensitivity. If we assume that the principal emission mechanism is IC scattering of $\mathrm{GeV}$ electrons off $\mathrm{CMB}$ photons, then the cluster magnetic field is constrained to be $\geqslant 0.1 \mu \mathrm{G}$. These low magnetic intensities would show that the magnetic field is far from equipartition (i.e., the energy in the magnetic field is different with respect to the electrons' energy). As pointed out by Petrosian \& Bykov (2008), this can happen if the sources generating the magnetic field and accelerating the electrons are not identical.

However, IC emission by relativistic electrons can be modeled as a power law in the $1-200 \mathrm{keV}$ energy regime (e.g., Nevalainen et al. 2004; Reimer et al. 2004). Thus, using XMM-Newton/ $\mathrm{XRT} /$ Chandra and BAT data, we are able to constrain, more robustly, the IC emission mechanism. With this approach, we confirm the detection and the flux of the hard component in the spectrum of A0754, but we are also able to prove (thanks to the resolution of XMM-Newton) that a single point-like object, 2MASS 09091372-0943047, located less than $2^{\prime}$ from the BAT centroid, accounts for the whole nonthermal emission. For the rest of the clusters, we are able to produce upper limits which are a factor of 5-10 lower than previously estimated. These limits, in turn, translate into a slightly larger intensity of the magnetic field which reduces the gap to Faraday rotation measurements (Kim et al. 1991; Clarke et al. 2001). If the cluster magnetic field is truly of the $\mu \mathrm{G}$ order, then the chances of detecting IC emission from clusters with the currently flying instruments become really small (Pfrommer \& Enßlin 2004). Indeed, the values of the predicted IC flux account for only less than $10 \%$ of the claimed nonthermal X-ray emission above $10 \mathrm{keV}$ when taking both primary- and secondary-generated electrons into account (see, e.g., Miniati et al. 2001). Recently, Pfrommer (2008), using high-resolution simulations of a sample of representative galaxy clusters, showed that the predicted IC flux for the Coma and Perseus clusters would be a factor of 50 lower than the detections claimed.
Our combined analysis thus puts tight constrains on the IC mechanism. However, IC emission is the process that most likely explains the claimed nonthermal emission, but not the only one. Hard X-ray flux from galaxy clusters can be interpreted as bremsstrahlung from a supra-thermal electron tail developed in the thermal electron distribution due to stochastic acceleration in the turbulent ICM (e.g., Enßlin et al. 1999; Petrosian 2001). In this modeling, the radio and the nonthermal X-ray fluxes are no longer strictly related and equipartition may apply. However, the nonthermal bremsstrahlung model requires a continuous input of energy in the ICM, which, as a consequence, will cause its temperature to increase. Thus, the nonthermal bremsstrahlung phase is likely to be short lived (Petrosian \& Bykov 2008).

\subsection{Structure Formation}

All the galaxy clusters detected by BAT, except perhaps A3571, are merging systems. Some, such as A0754, A2142, and A3266, experience violent merging due to encounters of subclusters with comparable masses. In the common scenarios of hierarchical structure formation (e.g., Miniati et al. 2000; Ryu \& Kang 2003), large systems evolve as the result of the merging of smaller structures. As reviewed in Dolag et al. (2008), cluster mergers generate internal shocks (Mach number less than 4), which provide most of the ICM gas heating (e.g., Quilis et al. 1998), and also likely convert a non-negligible fraction $(\leqslant 10 \%)$ of their power into CRs. The shocks primarily heat the ions because the kinetic energy of an ion entering the shock region is larger than that of an electron by their mass ratio (Takizawa 1999). Cosmological simulations have shown (e.g., Pfrommer et al. 2007) that, in the case of ongoing merger activity, the relative CR pressure (to the thermal ICM pressure) is greatly enhanced, up to $15-20 \%$, due to strong merger shock waves. This pressure is likely larger in the outskirts of the cluster because of the lower sound speed and the larger density of the ICM in the central region, which makes CR acceleration less efficient (Pfrommer et al. 2007).

Hot spots and cold fronts have been found in many merging clusters, thanks to the superior resolution of Chandra (e.g., Markevitch et al. 2000; Markevitch \& Vikhlinin 2001; Markevitch et al. 2003). Hydrodynamical simulations have highlighted that $\sim 1$ Gyr after the encounter of two clusters with comparable masses, postshock regions with high temperatures $(T \approx 10-20 \mathrm{keV}$ ) are formed (e.g., Takizawa 1999; Ritchie \& Thomas 2002). In the BAT sample, there is a clear correlation of gas temperature and merging activity. Indeed, A3571 and Perseus, which are in a late merging stage, display the lowest plasma temperatures among the clusters in our sample. INTEGRAL recently unveiled the presence of a hotter region $(T=12 \pm 2 \mathrm{keV})$, located south-west of the center of the Coma cluster (Eckert et al. 2008a). These findings highlight the important role of merging shocks in the heating of the ICM.

\subsection{Clusters Statistics}

The serendipitous character of the BAT survey allowed us to determine, for the first time above $10 \mathrm{keV}$, the $\log N-\log S$ and luminosity function distributions of galaxy clusters. Both are in very good agreement with previous studies. The $\log N-\log$ $S$ highlights that the clusters BAT detects produce a negligible fraction $(\sim 0.1 \%)$ of the X-ray background emission, but they represent a sizeable population (5-10\%) with respect to the local 
AGN. The BAT $\log N-\log S$ shows that future instruments with a sensitivity 10 or 100 times better than BAT (above $15 \mathrm{keV}$ ) will detect clusters at densities of $\sim 0.01 \mathrm{deg}^{-2}$ and $\sim 0.24 \mathrm{deg}^{-2}$, respectively.

The BAT luminosity distribution allowed us to determine that the volume emissivity of galaxy clusters is $W(>2 \times$ $\left.10^{43} \mathrm{erg} \mathrm{s}^{-1}\right)=2.38 \times 10^{37} \mathrm{erg} \mathrm{s}^{-1} \mathrm{Mpc}^{-3}$. Above the same limiting luminosity, Sazonov et al. (2007) derived that the volume emissivity of the local $\mathrm{AGN}$ is $W_{\mathrm{AGN}}=14.1 \times$ $10^{37} \mathrm{erg} \mathrm{s}^{-1} \mathrm{Mpc}^{-3}$. Thus, above $2 \times 10^{43} \mathrm{erg} \mathrm{s}^{-1}$, the cluster volume emissivity is $20 \%$ of that of AGN. Integrating the luminosity functions into lower luminosity (e.g., $10^{41} \mathrm{erg} \mathrm{s}^{-1}$ ) changes this fraction to $\sim 10 \%$. This change is due to the fact that at low luminosity, the AGN luminosity function is steeper than the cluster luminosity function (e.g., Sazonov et al. 2007; Böhringer et al. 2002).

\subsection{Future Prospects}

The study of nonthermal processes in clusters of galaxies requires a multiwavelength approach. The ongoing Swift/BAT survey will likely comprise of up to 30 clusters if an all-sky sensitivity of $0.5 \mathrm{mCrab}$ is reached and it will improve the $\mathrm{S} / \mathrm{N}$ for the spectra of the clusters presented here. Ultimately, major progress is expected with the launch of Simbol- $\mathrm{X}^{13}$, $\mathrm{XEUS}^{14}$, NUSTAR ${ }^{15}$, and NeXT ${ }^{16}$. Indeed, their sensitivities and spectro-imaging capabilities up to high energies $(80 \mathrm{keV}$ and beyond) will provide new and better constraints on the hard $\mathrm{X}$-ray emission.

The future generation of radio arrays combined with highenergy observations will allow us to shed some light on the energetics of relativistic particles, the nature and frequency of acceleration processes, and the strength and structure of magnetic fields. As we already discussed, this astrophysical information has strong cosmological implications. The Long Wavelength Array ${ }^{17}$ (LWA), the Low Frequency Array ${ }^{18}$ (LOFAR), and ultimately the Square Kilometre Array ${ }^{19}$ (SKA) will operate over a critical radio frequency range to detect relativistic plasma in large-scale structures and clusters in a sensitive way. The advance in sensitivity and resolution will increase the statistics of known radio halos and radio relics at different redshifts. The correlation of sensitive X-ray and radio detections will be particularly important (e.g., Enßlin \& Röttgering 2002). At the same time, thanks to the high angular and spectral resolution, the Faraday rotation studies will significantly improve, yielding a better determination of the cluster magnetic field.

Much attention is directed toward the Gamma-Ray Large Area Space Telescope ${ }^{20}$ (GLAST) which, with an unprecedented sensitivity, spatial resolution, and dynamic range at $\mathrm{GeV}$ energies, will shed light on the origin of the extragalactic $\gamma$-ray background. Galaxy clusters and shocks from structure formations are natural candidates for explaining part of this diffuse emission (e.g., Dermer 2007, and references therein). All the BAT clusters are good candidates for GLAST since they are nearby and are mergers. Indeed, in merging systems, part of

\footnotetext{
13 http://www.asdc.asi.it/simbol-x/

14 http://www.rssd.esa.int/index.php?project=xeus

15 http://www.nustar.caltech.edu/

$16 \mathrm{http}: / /$ www.astro.isas.ac.jp/future/NeXT/

17 http://lwa.unm.edu

18 http://www.lofar.org/

19 http://www.skatelescope.org

20 http://www-glast.stanford.edu
}

the internal shocks' energy is very likely converted into CRs' acceleration (Dolag et al. 2008). As pointed out by Pfrommer et al. (2008), above $100 \mathrm{MeV}$, the cluster emission will likely be dominated by pion decay $\gamma$-rays even though a contribution from nonthermal bremsstrahlung and IC emission of secondary electrons is expected. This will provide unique information about the hadron component of CRs, which is not included in estimates of $\mathrm{CR}$ pressure based only on the observations discussed above concerning electrons and magnetic field. Since CR protons' loss time is long, the $\pi^{0}$-bump detection would prove that hadrons have been confined in the ICM for as long as the Hubble time (e.g., Berezinsky et al. 1997). Stringent constraints on the CRs' content in the ICM are fundamental for the future space missions which will use galaxy clusters to constrain and understand the nature of Dark Energy (e.g., eROSITA ${ }^{21}$ ).

\section{CONCLUSIONS}

BAT is the first instrument to detect an all-sky sample of galaxy clusters above $15 \mathrm{keV}^{22}$. The BAT energy range (15$200 \mathrm{keV}$ ) is the best one to investigate the presence of nonthermal emission, whose detection has so far remained controversial. The results of our investigation can be summarized as follows.

1. Perseus is the only cluster among the 10 BAT objects which displays a high-energy nonthermal component that extends up to $200 \mathrm{keV}$. It is very likely that the central AGN NGC 1275 is responsible for such emission. This claim is supported by the following evidence: (1) the variability seen with BeppoSAX (Nevalainen et al. 2004), (2) the XMMNewton spectral analysis (Churazov et al. 2003), and (3) our combined BAT-XRT-XMM-Newton analysis, which shows that the nucleus has a typical AGN spectrum.

2. The BAT spectra of the remaining nine galaxy clusters are well fitted by a simple thermal model that constrains the nonthermal flux to be below $1 \mathrm{mCrab}$ in the $50-100 \mathrm{keV}$ band.

3. Assuming that IC scattering is the main mechanism at work for producing nonthermal high-energy flux, it is possible to estimate the magnetic field using radio data and the upper limits derived above. We obtain that, in all the BAT clusters, the (average) magnetic field is greater than $0.1 \mu \mathrm{G}$. These (rather uncertain) values are in disagreement (if the magnetic field intensities are close to the lower limits) with the, also uncertain, Faraday rotation measurements, which show that the magnetic field is in the $\sim \mu \mathrm{G}$ range. Our low magnetic field values would imply that the magnetic field is far from equipartition.

4. The stacked spectrum of the BAT clusters (except Perseus and Coma) confirms once again the absence of any nonthermal high-energy component. The $\sim 56 \mathrm{Ms}$ stacked spectrum constrains any nonthermal flux to be below $0.3 \mathrm{mCrab}$ (or $1.9 \times 10^{-12} \mathrm{erg} \mathrm{cm}^{-2} \mathrm{~s}^{-1}$ ) in the $50-100 \mathrm{keV}$ band.

5. Using Swift/XRT, XMM-Newton, and Chandra, in addition to BAT data, we were able to produce X-ray cluster spectra that extend more than three decades in energy $(0.5-$ $50 \mathrm{keV}$ ). In all cases except Perseus and A0754, the broadband X-ray spectrum is well approximated by a singletemperature thermal model. These spectra allowed us to

\footnotetext{
21 http://www.mpe.mpg.de/projects.html\#erosita

22 We are aware of an independent work (T. Okajima 2008, in preparation) based on an alternative analysis of BAT survey data, which reaches conclusions consistent with this analysis.
} 
put constraints on the IC emission mechanism, which are more than a factor of 5 lower than those derived using BAT data alone. This would, in turn, imply a larger intensity of the magnetic field. For both Perseus and A0754, an additional power-law component is statistically required, but there is evidence to confirm that two X-ray point sources (NGC 1275 and 2MASS 09091372-0943047) account for the total nonthermal emission.

6. The cluster centroid shift in different wavebands, the morphology, and the complex temperature maps (available in the literature) show that eight out of 10 clusters are in the middle of a major merging phase. Shocks, which are revealed by XMM-Newton and Chandra images, actively heat the ICM as the BAT high temperatures testify. The BAT observations and limits on the nonthermal emissions can help to calibrate the large-scale structure formation simulations focusing, in particular, on the treatment of nonthermal particle emission and cooling.

(a) We have produced the first cluster source count (also known as $\log N-\log S$ ) distribution above $15 \mathrm{keV}$. This shows that, at the limiting fluxes sampled by BAT, the surface density of clusters is $\sim 5 \%$ of that of AGNs. Moreover, we find that the contribution of clusters to the CXB is of order $\sim 0.1 \%$ in the $15-55 \mathrm{keV}$ band. The BAT $\log N-\log S$ can be used to predict the cluster surface density for future hard X-ray instruments.

(b) The X-ray luminosity function of the BAT clusters, the first derived above $15 \mathrm{keV}$, is in excellent agreement with the ROSAT luminosity function derived in the $0.1-2.4 \mathrm{keV}$ band.

M.A. acknowledges funding from the DFG Leibniz-Prize (HA 1850/28-1). P.R. is supported by the Pappalardo Postdoctoral Fellowship in Physics at MIT. N.C. was partially supported by a NASA grant NNX07AV03G. M.A. and P.R. acknowledge Balú for his incomparable constant enthusiasm. We thank T. Okajima for providing a copy of his manuscript before submission and for interesting discussions. The anonymous referee is acknowledged for his helpful comments, which improved the manuscript. This research has made use of the NASA/IPAC Extragalactic Database, which is operated by the Jet Propulsion Laboratory, of data obtained from the High Energy Astrophysics Science Archive Research Center provided by NASA's Goddard Space Flight Center, of the SIMBAD Astronomical Database, which is operated by the Centre de Données astronomiques de Strasbourg, and of the ROSAT All Sky Survey maintained by the Max Planck Institut für Extraterrestrische Physik.

Facilities: Swift (BAT/XRT), XMM-Newton, Chandra.

\section{REFERENCES}

Ajello, M., Greiner, J., Kanbach, G., Rau, A., Strong, A. W., \& Kennea, J. A. 2008a, ApJ, 678, 102

Ajello, M., Greiner, J., Sato, G., \& Kanbach, G. 2008b, ApJ, accepted

Ajello, M., et al. 2008c, ApJ, 673, 96

Anders, E., \& Grevesse, N. 1989, Geochim. Cosmochim. Acta, 53, 197

Ando, S., \& Nagai, D. 2008, MNRAS, 385, 2243

Arnaud, M., \& Evrard, A. E. 1999, MNRAS, 305, 631

Arnaud, M., et al. 2001, A\&A, 365, L67

Avni, Y. 1976, ApJ, 210, 642

Bacchi, M., Feretti, L., Giovannini, G., \& Govoni, F. 2003, A\&A, 400, 465

Barthelmy, S. D., et al. 2005, Space Sci. Rev., 120, 143

Berezinsky, V. S., Blasi, P., \& Ptuskin, V. S. 1997, ApJ, 487, 529

Bird, A. J., et al. 2006, ApJ, 636, 765

Blanton, E. L., Sarazin, C. L., \& McNamara, B. R. 2003, ApJ, 585, 227
Blasi, P., Gabici, S., \& Brunetti, G. 2007, arXiv:astro-ph/0701545 Böhringer, H., et al. 2001, A\&A, 369, 826

Böhringer, H., et al. 2002, ApJ, 566, 93

Bonamente, M., Lieu, R., \& Mittaz, J. P. D. 2001, ApJ, 561, L63

Bowyer, S., Berghöfer, T. W., \& Korpela, E. J. 1999, ApJ, 526, 592

Brown, D. L., \& Burns, J. O. 1991, AJ, 102, 1917

Brunetti, G., Blasi, P., Cassano, R., \& Gabici, S. 2004, MNRAS, 350, 1174

Burenin, R. A., Vikhlinin, A., Hornstrup, A., Ebeling, H., Quintana, H., \& Mescheryakov, A. 2007, ApJS, 172, 561

Bykov, A. M., Bloemen, H., \& Uvarov, Y. A. 2000, A\&A, 362, 886

Catura, R. C., Fisher, P. C., Johnson, H. M., \& Meyerott, A. J. 1972, ApJ, 177, L1

Cavagnolo, K. W., Donahue, M., Voit, M., \& Sun, M. 2008, ApJ, 682, 821

Chen, Y., Reiprich, T. H., Böhringer, H., Ikebe, Y., \& Zhang, Y.-Y. 2007, A\&A, 466, 805

Churazov, E., Forman, W., Jones, C., \& Böhringer, H. 2003, ApJ, 590, 225

Churazov, E., Forman, W., Vikhlinin, A., Tremaine, S., Gerhard, O., \& Jones, C. 2008, MNRAS, 388, 1062

Clarke, T. E., Blanton, E. L., \& Sarazin, C. L. 2004, ApJ, 616, 178

Clarke, T. E., Kronberg, P. P., \& Böhringer, H. 2001, ApJ, 547, L111

Colafrancesco, S., Marchegiani, P., \& Perola, G. C. 2005, A\&A, 443, 1

Condon, J. J., Cotton, W. D., Greisen, E. W., Yin, Q. F., Perley, R. A., Taylor, G. B., \& Broderick, J. J. 1998, AJ, 115, 1693

Condon, J. J., Griffith, M. R., \& Wright, A. E. 1993, AJ, 106, 1095

Dar, A., \& Shaviv, N. J. 1995, Phys. Rev. Lett., 75, 3052

De Grandi, S., \& Molendi, S. 1999, ApJ, 527, L25

De Grandi, S., \& Molendi, S. 2002, ApJ, 567, 163

de Plaa, J., et al. 2006, A\&A, 452, 397

Dennison, B. 1980, ApJ, 239, L93

Dermer, C. D. 2007, ApJ, 659, 958

Dolag, K., Bykov, A. M., \& Diaferio, A. 2008, Space Sci. Rev., 134, 311

Durret, F., Slezak, E., Lieu, R., Dos Santos, S., \& Bonamente, M. 2002, A\&A, 390, 397

Ebisawa, K., Bourban, G., Bodaghee, A., Mowlavi, N., \& Courvoisier, T. J.-L. 2003, A\&A, 411, L59

Eckert, D., Produit, N., Neronov, A., \& Courvoisier, T. J. 2008a, A\&A, 479, 27

Eckert, D., Produit, N., Paltani, S., Neronov, A., \& Courvoisier, T. J.-L. 2008b, A\&A, 479, 27

Edge, A. C., Stewart, G. C., \& Fabian, A. C. 1992, MNRAS, 258, 177

Ensslin, T. A., Biermann, P. L., Kronberg, P. P., \& Wu, X.-P. 1997, ApJ, 477, 560

Enßlin, T. A., Lieu, R., \& Biermann, P. L. 1999, A\&A, 344, 409

Enßlin, T. A., \& Röttgering, H. 2002, A\&A, 396, 83

Ettori, S., Fabian, A. C., \& White, D. A. 1998, MNRAS, 300, 837

Fabian, A. C., Pringle, J. E., \& Rees, M. J. 1976, Nature, 263, 301

Felten, J. E., Gould, R. J., Stein, W. A., \& Woolf, N. J. 1966, ApJ, 146, 955

Feretti, L., \& Giovannini, G. 1998, in Untangling Coma Berenices: A New Vision of an Old Cluster, ed. A. Mazure, F. Casoli, F. Durret, \& D. Gerbal (Singapore: World Scientific), 123

Feretti, L., \& Giovannini, G. 2007, arXiv:astro-ph/0703494

Feretti, L., Giovannini, G., \& Bohringer, H. 1997, New Astron., 2, 501

Finoguenov, A., Briel, U. G., Henry, J. P., Gavazzi, G., Iglesias-Paramo, J., \& Boselli, A. 2004, A\&A, 419, 47

Finoguenov, A., Henriksen, M. J., Miniati, F., Briel, U. G., \& Jones, C. 2006, ApJ, 643, 790

Forman, W., Kellogg, E., Gursky, H., Tananbaum, H., \& Giacconi, R. 1972, ApJ, 178, 309

Fritz, G., Davidsen, A., Meekins, J. F., \& Friedman, H. 1971, ApJ, 164, L81

Fujita, Y., et al. 2008, arXiv:0806.3747

Fujita, Y., Kohri, K., Yamazaki, R., \& Kino, M. 2007, ApJ, 663, L61

Fujita, Y., Takizawa, M., \& Sarazin, C. L. 2003, ApJ, 584, 190

Fusco-Femiano, R., dal Fiume, D., Feretti, L., Giovannini, G., Grandi, P., Matt, G., Molendi, S., \& Santangelo, A. 1999, ApJ, 513, L21

Fusco-Femiano, R., Landi, R., \& Orlandini, M. 2007, ApJ, 654, L9

Fusco-Femiano, R., Orlandini, M., Brunetti, G., Feretti, L., Giovannini, G., Grandi, P., \& Setti, G. 2004, ApJ, 602, L73

Fusco-Femiano, R., Orlandini, M., De Grandi, S., Molendi, S., Feretti, L., Giovannini, G., Bacchi, M., \& Govoni, F. 2003, A\&A, 398, 441

Gehrels, N. 1986, ApJ, 303, 336

Gehrels, N., et al. 2004, ApJ, 611, 1005

Giacconi, R., Murray, S., Gursky, H., Kellogg, E., Schreier, E., \& Tananbaum, H. 1972, ApJ, 178, 281

Giovannini, G., \& Feretti, L. 2000, New Astron., 5, 335

Giovannini, G., Feretti, L., Venturi, T., Kim, K.-T., \& Kronberg, P. P. 1993, ApJ, 406, 399

Gisler, G. R., \& Miley, G. K. 1979, A\&A, 76, 109

Gitti, M., Brunetti, G., \& Setti, G. 2002, A\&A, 386, 456 
Goldshmidt, O., \& Rephaeli, Y. 1993, ApJ, 411, 518

Gruber, D., \& Rephaeli, Y. 2002, ApJ, 565, 877

Harris, D. E., Bahcall, N. A., \& Strom, R. G. 1977, A\&A, 60, 27

Harris, D. E., \& Miley, G. K. 1978, A\&AS, 34, 117

Harris, D. E., \& Romanishin, W. 1974, ApJ, 188, 209

Henriksen, M. J., \& Markevitch, M. L. 1996, ApJ, 466, L79

Henry, J. P., \& Briel, U. G. 1995, ApJ, 443, L9

Henry, J. P., \& Briel, U. G. 1996, ApJ, 472, 137

Henry, J. P., Finoguenov, A., \& Briel, U. G. 2004, ApJ, 615, 181

Ikebe, Y., Reiprich, T. H., Böhringer, H., Tanaka, Y., \& Kitayama, T. 2002, A\&A, 383, 773

Inoue, S., Aharonian, F. A., \& Sugiyama, N. 2005, ApJ, 628, L9

Johnston, M. D., Bradt, H. V., Doxsey, R. E., Marshall, F. E., Schwartz, D. A., \& Margon, B. 1981, ApJ, 245, 799

Jones, B. B., \& Finlay, E. A. 1974, Aust. J. Phys., 27, 687

Jones, L. R., Scharf, C., Ebeling, H., Perlman, E., Wegner, G., Malkan, M., \& Horner, D. 1998, ApJ, 495, 100

Kaastra, J. S., et al. 2008, Space Sci. Rev., 134, 155

Katz, J. I. 1976, ApJ, 207, 25

Kempner, J. C., \& Sarazin, C. L. 2001, ApJ, 548, 639

Kennea, J. A., et al. 2005, The Astronomer's Telegram, 677, 1

Kim, K.-T., Kronberg, P. P., \& Tribble, P. C. 1991, ApJ, 379, 80

Lieu, R., Mittaz, J. P. D., Bowyer, S., Lockman, F. J., Hwang, C.-Y., \& Schmitt, J. H. M. M. 1996, ApJ, 458, L5

Lumb, D. H., Warwick, R. S., Page, M., \& De Luca, A. 2002, A\&A, 389, 93

Lutovinov, A. A., Vikhlinin, A., Churazov, E. M., Revnivtsev, M. G., \& Sunyaev, R. A. 2008, Proc. 6th INTEGRAL Workshop " The Obscured Universe”, ESA SP-622, 241

Markevitch, M. 1998, ApJ, 504, 27

Markevitch, M., Forman, W. R., Sarazin, C. L., \& Vikhlinin, A. 1998, ApJ, 503, 77

Markevitch, M., \& Vikhlinin, A. 2001, ApJ, 563, 95

Markevitch, M., et al. 2000, ApJ, 541, 542

Markevitch, M., et al. 2003, ApJ, 586, L19

Markevitch, M. L., Sarazin, C. L., \& Irwin, J. A. 1996, ApJ, 472, L17

Markwardt, C. B., Tueller, J., Skinner, G. K., Gehrels, N., Barthelmy, S. D., \& Mushotzky, R. F. 2005, ApJ, 633, L77

Mills, B. Y., Slee, O. B., \& Hill, E. R. 1960, Aust. J. Phys., 13, 676

Miniati, F., Jones, T. W., Kang, H., \& Ryu, D. 2001, ApJ, 562, 233

Miniati, F., Ryu, D., Kang, H., Jones, T. W., Cen, R., \& Ostriker, J. P. 2000, ApJ, 542, 608

Miralda Escude, J., \& Babul, A. 1995, ApJ, 449, 18

Molendi, S., \& de Grandi, S. 1999, A\&A, 351, L41

Molendi, S., de Grandi, S., Fusco-Femiano, R., Colafrancesco, S., Fiore, F., Nesci, R., \& Tamburelli, F. 1999, ApJ, 525, L73

Molendi, S., \& Gastaldello, F. 2008, arXiv:0807.2653

Nagai, D., Vikhlinin, A., \& Kravtsov, A. V. 2007, ApJ, 655, 98

Nevalainen, J., Kaastra, J., Parmar, A. N., Markevitch, M., Oosterbroek, T. Colafrancesco, S., \& Mazzotta, P. 2001, A\&A, 369, 459

Nevalainen, J., Oosterbroek, T., Bonamente, M., \& Colafrancesco, S. 2004, ApJ, 608,166

O'Hara, T. B., Mohr, J. J., \& Guerrero, M. A. 2004, ApJ, 604, 604

Ota, N., Kitayama, T., Masai, K., \& Mitsuda, K. 2006, ApJ, 640, 673

Peres, C. B., Fabian, A. C., Edge, A. C., Allen, S. W., Johnstone, R. M., \& White, D. A. 1998, MNRAS, 298, 416

Perkins, J. S., et al. 2006, ApJ, 644, 148

Petrosian, V. 2001, ApJ, 557, 560

Petrosian, V., \& Bykov, A. M. 2008, Space Sci. Rev., 134, 207

Pfrommer, C. 2008, MNRAS, 385, 1242

Pfrommer, C., \& Enßlin, T. A. 2004, A\&A, 413, 17

Pfrommer, C., Enßlin, T. A., \& Springel, V. 2008, MNRAS, 385, 1211

Pfrommer, C., Enßlin, T. A., Springel, V., Jubelgas, M., \& Dolag, K. 2007, MNRAS, 378,385
Primini, F. A., et al. 1981, ApJ, 243, L13

Quilis, V., Ibanez, J. M. A., \& Saez, D. 1998, ApJ, 502, 518

Read, A. M., \& Ponman, T. J. 2003, A\&A, 409, 395

Reimer, A., Reimer, O., Schlickeiser, R., \& Iyudin, A. 2004, A\&A, 424, 773

Reimer, O., Pohl, M., Sreekumar, P., \& Mattox, J. R. 2003, ApJ, 588, 155

Renaud, M., Bélanger, G., Paul, J., Lebrun, F., \& Terrier, R. 2006a, A\&A, 453, L5

Renaud, M., Gros, A., Lebrun, F., Terrier, R., Goldwurm, A., Reynolds, S., \& Kalemci, E. 2006b, A\&A, 456, 389

Rephaeli, Y. 1979, ApJ, 227, 364

Rephaeli, Y. 2001, in AIP Conf. Ser. 588, High Energy Gamma-Ray Astronomy, Int. Symp., ed. F. A. Aharonian \& H. J. Völk (New York: AIP), 427

Rephaeli, Y., \& Gruber, D. 2002, ApJ, 579, 587

Rephaeli, Y., Nevalainen, J., Ohashi, T., \& Bykov, A. M. 2008, Space Sci. Rev., 134,71

Revnivtsev, M., Sazonov, S., Jahoda, K., \& Gilfanov, M. 2004, A\&A, 418, 927

Ritchie, B. W., \& Thomas, P. A. 2002, MNRAS, 329, 675

Robertson, J. G., \& Roach, G. J. 1990, MNRAS, 247, 387

Rossetti, M., \& Molendi, S. 2004, A\&A, 414, L41

Rossetti, M., \& Molendi, S. 2007, arXiv:astro-ph/0702417

Ryu, D., \& Kang, H. 2003, J. Korean Astron. Soc., 36, 105

Sanders, J. S., \& Fabian, A. C. 2007, MNRAS, 381, 1381

Sanders, J. S., Fabian, A. C., Allen, S. W., \& Schmidt, R. W. 2004, MNRAS, 349,952

Sanders, J. S., Fabian, A. C., \& Dunn, R. J. H. 2005, MNRAS, 360, 133

Sanderson, A. J. R., Ponman, T. J., \& O'Sullivan, E. 2006, MNRAS, 372,1496

Sarazin, C. L. 1988, in Cambridge Astrophysics Series, X-ray Emission from Clusters of Galaxies (Cambridge: Cambridge Univ. Press)

Sarazin, C. L. 1999, ApJ, 520, 529

Sarazin, C. L., \& Kempner, J. C. 2000, ApJ, 533, 73

Sarazin, C. L., Wise, M. W., \& Markevitch, M. L. 1998a, ApJ, 498, 606

Sarazin, C. L., Wise, M. W., \& Markevitch, M. L. 1998b, ApJ, 498, 606

Sauvageot, J. L., Belsole, E., \& Pratt, G. W. 2005, A\&A, 444, 673

Sazonov, S., Revnivtsev, M., Krivonos, R., Churazov, E., \& Sunyaev, R. 2007, A\&A, 462, 57

Slee, O. B. 1977, Aust. J. Phys. Astrophys. Suppl., 43, 1

Slee, O. B., \& Higgins, C. S. 1975, Aust. J. Phys. Astrophys. Suppl., 36, 1

Smith, R. K., Brickhouse, N. S., Liedahl, D. A., \& Raymond, J. C. 2001, ApJ, 556, L91

Stephen, J. B., et al. 2006, A\&A, 445, 869

Takizawa, M. 1999, ApJ, 520, 514

Taylor, G. B., Barton, E. J., \& Ge, J. 1994, AJ, 107, 1942

Thierbach, M., Klein, U., \& Wielebinski, R. 2003, A\&A, 397, 53

Timokhin, A. N., Aharonian, F. A., \& Neronov, A. Y. 2004, A\&A, 417, 391

Tueller, J., et al. 2005a, The Astronomer's Telegram, 668, 1

Tueller, J., et al. 2005b, The Astronomer's Telegram, 669, 1

Valinia, A., Henriksen, M. J., Loewenstein, M., Roettiger, K., Mushotzky, R. F., \& Madejski, G. 1999, ApJ, 515, 42

Venturi, T., Bardelli, S., Zagaria, M., Prandoni, I., \& Morganti, R. 2002, A\&A, 385,39

Vikhlinin, A., McNamara, B. R., Forman, W., Jones, C., Quintana, H., \& Hornstrup, A. 1998, ApJ, 498, L21

Voges, W., et al. 1999, A\&A, 349, 389

Watanabe, M., Yamashita, K., Furuzawa, A., Kunieda, H., \& Tawara, Y. 2001a PASJ, 53, 605

Watanabe, M., Yamashita, K., Furuzawa, A., Kunieda, H., \& Tawara, Y. 2001b, PASJ, 53, 605

Werner, N., Kaastra, J. S., Takei, Y., Lieu, R., Vink, J., \& Tamura, T. 2007, A\&A, 468, 849

White, R. E., III, Day, C. S. R., Hatsukade, I., \& Hughes, J. P. 1994, ApJ, 433, 583

Willson, M. A. G. 1970, MNRAS, 151, 